\title{
Some fractional calculus findings associated with the incomplete $I$-functions
}

\author{
Kamlesh Jangid', Sanjay Bhatter ${ }^{2}$, Sapna Meena², Dumitru Baleanu ${ }^{3,4,5}$, Maysaa Al Qurashi ${ }^{6}$ and \\ Sunil Dutt Purohit ${ }^{*}$ (D)
}

"Correspondence:
sunil_a_purohit@yahoo.com
'Department of HEAS
(Mathematics), Rajasthan Technical
University, Kota, India
Full list of author information is
available at the end of the article

\begin{abstract}
In this article, several interesting properties of the incomplete I-functions associated with the Marichev-Saigo-Maeda (MSM) fractional operators are studied and investigated. It is presented that the order of the incomplete I-functions increases about the utilization of the above-mentioned operators toward the power multiple of the incomplete I-functions. Further, the Caputo-type MSM fractional order differentiation for the incomplete /-functions is studied and investigated. Saigo, Riemann-Liouville, and Erdélyi-Kober fractional operators are also discussed as specific cases.
\end{abstract}

MSC: 33B20; 33E20; 26A33

Keywords: Fractional calculus operators; Incomplete gamma functions; Incomplete I-functions; Mellin-Barnes type contour

\section{Introduction and preliminaries}

Recently, Jangid et al. [11] defined a new family of incomplete $I$-functions ${ }^{\gamma} I_{p, q}^{m, n}(z)$ and ${ }^{\Gamma} I_{p, q}^{m, n}(z)$. Incomplete $I$-functions are the natural generalization of the $I$-function defined by Rathie [27]. They are an expansion of a familiar Fox's $H$-function [6] and many other special functions. Fractional calculus for the variety of special functions is being widely used in mathematical modeling, statistical distribution, wireless communication, and engineering sciences (see $[1,3-5,7-10,12,14-16,18,20-23,28,31-34])$. The incomplete $I$-functions in the form of Mellin-Barnes type contour integrals are defined as

$$
\begin{aligned}
& { }^{\gamma} I_{p, q}^{m, n}(z)={ }^{\gamma} I_{p, q}^{m, n}\left[z \mid \begin{array}{c}
\left(a_{1}, \sigma_{1} ; A_{1}: x\right),\left(a_{2}, \sigma_{2} ; A_{2}\right), \ldots,\left(a_{p}, \sigma_{p} ; A_{p}\right) \\
\left(b_{1}, \rho_{1} ; B_{1}\right), \ldots,\left(b_{q}, \rho_{q} ; B_{q}\right)
\end{array}\right] \\
& ={ }^{\gamma} I_{p, q}^{m, n}\left[z \mid \begin{array}{c}
\left(a_{1}, \sigma_{1} ; A_{1}: x\right),\left(a_{j}, \sigma_{j} ; A_{j}\right)_{2, p} \\
\left(b_{j}, \rho_{j} ; B_{j}\right)_{1, q}
\end{array}\right] \\
& =\frac{1}{2 \pi i} \int_{\$} \phi(s, x) z^{s} d s
\end{aligned}
$$

(c) The Author(s) 2020. This article is licensed under a Creative Commons Attribution 4.0 International License, which permits use, sharing, adaptation, distribution and reproduction in any medium or format, as long as you give appropriate credit to the original author(s) and the source, provide a link to the Creative Commons licence, and indicate if changes were made. The images or other third party material in this article are included in the article's Creative Commons licence, unless indicated otherwise in a credit line to the material. If material is not included in the article's Creative Commons licence and your intended use is not permitted by statutory regulation or exceeds the permitted use, you will need to obtain permission directly from the copyright holder. To view a copy of this licence, visit http://creativecommons.org/licenses/by/4.0/. 
and

$$
\begin{aligned}
\Gamma I_{p, q}^{m, n}(z) & =\Gamma_{p, q}^{m, n}\left[z \mid \begin{array}{c}
\left(a_{1}, \sigma_{1} ; A_{1}: x\right),\left(a_{2}, \sigma_{2} ; A_{2}\right), \ldots,\left(a_{p}, \sigma_{p} ; A_{p}\right) \\
\left(b_{1}, \rho_{1} ; B_{1}\right), \ldots,\left(b_{q}, \rho_{q} ; B_{q}\right)
\end{array}\right] \\
& =\Gamma I_{p, q}^{m, n}\left[z \mid \begin{array}{c}
\left(a_{1}, \sigma_{1} ; A_{1}: x\right),\left(a_{j}, \sigma_{j} ; A_{j}\right)_{2, p} \\
\left(b_{j}, \rho_{j} ; B_{j}\right)_{1, q}
\end{array}\right] \\
& =\frac{1}{2 \pi i} \int_{\$} \Phi(s, x) z^{s} d s
\end{aligned}
$$

for all $z \neq 0$, where

$$
\phi(s, x)=\frac{\left\{\gamma\left(1-a_{1}+\sigma_{1} s, x\right)\right\}^{A_{1}} \prod_{j=1}^{m}\left\{\Gamma\left(b_{j}-\rho_{j} s\right)\right\}^{B_{j}} \prod_{j=2}^{n}\left\{\Gamma\left(1-a_{j}+\sigma_{j} s\right)\right\}^{A_{j}}}{\prod_{j=n+1}^{p}\left\{\Gamma\left(a_{j}-\sigma_{j} s\right)\right\}^{A_{j}} \prod_{j=m+1}^{q}\left\{\Gamma\left(1-b_{j}+\rho_{j} s\right)\right\}^{B_{j}}}
$$

and

$$
\Phi(s, x)=\frac{\left\{\Gamma\left(1-a_{1}+\sigma_{1} s, x\right)\right\}^{A_{1}} \prod_{j=1}^{m}\left\{\Gamma\left(b_{j}-\rho_{j} s\right)\right\}^{B_{j}} \prod_{j=2}^{n}\left\{\Gamma\left(1-a_{j}+\sigma_{j} s\right)\right\}^{A_{j}}}{\prod_{j=n+1}^{p}\left\{\Gamma\left(a_{j}-\sigma_{j} s\right)\right\}^{A_{j}} \prod_{j=m+1}^{q}\left\{\Gamma\left(1-b_{j}+\rho_{j} s\right)\right\}^{B_{j}}},
$$

where $\gamma(\cdot, x)$ and $\Gamma(\cdot, x)$ are the lower and upper incomplete gamma functions defined in (6) and (7). The incomplete $I$-functions ${ }^{\gamma} I_{p, q}^{m, n}(z)$ and ${ }^{\Gamma} I_{p, q}^{m, n}(z)$ exist for all $x \geq 0$ under the same contour and conditions as stated in Rathie [27]. The incomplete $I$-functions fulfill the following relation (known as decomposition formula):

$$
\gamma_{p, q}^{m, n}(z)+{ }^{\Gamma} I_{p, q}^{m, n}(z)=I_{p, q}^{m, n}(z)
$$

for the familiar $I$-function given by Rathie [27]. Additionally, if we set $x=0$ in (2), then we obtain the $I$-function [27].

In the sequence we shall use the following statements and descriptions.

The familiar lower and upper incomplete gamma functions $\gamma(s, x)$ and $\Gamma(s, x)$, respectively, are laid out as follows:

$$
\gamma(s, x)=\int_{0}^{x} y^{s-1} e^{-y} d y \quad(\Re(s)>0 ; x \geqq 0)
$$

and

$$
\Gamma(s, x)=\int_{x}^{\infty} y^{s-1} e^{-y} d y \quad(x \geqq 0 ; \Re(s)>0 \text { when } x=0) .
$$

These incomplete gamma functions fulfill the following relation (known as decomposition formula):

$$
\gamma(s, x)+\Gamma(s, x)=\Gamma(s) \quad(\Re(s)>0) .
$$

In this article, several fractional calculus results associated with the incomplete $I$ functions are obtained. For $\sigma, \sigma^{\prime}, \rho, \rho^{\prime}, \eta \in \mathbb{C}$ and $x>0$ with $\Re(\eta)>0$, the left- and right- 
hand sided MSM fractional integral operators (see [19]) are defined as

$$
\begin{aligned}
\left(\mathcal{I}_{0+}^{\sigma, \sigma^{\prime}, \rho, \rho^{\prime}, \eta} f\right)(x)= & \frac{x^{-\sigma}}{\Gamma(\eta)} \int_{0}^{x}(x-y)^{\eta-1} y^{-\sigma^{\prime}} \\
& \times F_{3}\left(\sigma, \sigma^{\prime}, \rho, \rho^{\prime} ; \eta ; 1-\frac{y}{x}, 1-\frac{x}{y}\right) f(y) d y
\end{aligned}
$$

and

$$
\begin{aligned}
\left(\mathcal{I}_{-}^{\sigma, \sigma^{\prime}, \rho, \rho^{\prime}, \eta} f\right)(x) & =\frac{x^{-\sigma^{\prime}}}{\Gamma(\eta)} \int_{x}^{\infty}(y-x)^{\eta-1} y^{-\sigma} \\
& \times F_{3}\left(\sigma, \sigma^{\prime}, \rho, \rho^{\prime} ; \eta ; 1-\frac{x}{y}, 1-\frac{y}{x}\right) f(y) d y,
\end{aligned}
$$

respectively. The left- and right-hand sided MSM fractional differential operators (see [30]) are defined as

$$
\left(\mathcal{D}_{0+}^{\sigma, \sigma^{\prime}, \rho, \rho^{\prime}, \eta} f\right)(x)=\left(\frac{d}{d x}\right)^{\alpha}\left(\mathcal{I}_{0+}^{-\sigma^{\prime},-\sigma,-\rho^{\prime}+\alpha,-\rho,-\eta+\alpha} f\right)(x)
$$

and

$$
\left(\mathcal{D}_{-}^{\sigma, \sigma^{\prime}, \rho, \rho^{\prime}, \eta} f\right)(x)=\left(-\frac{d}{d x}\right)^{\alpha}\left(\mathcal{I}_{-}^{-\sigma^{\prime},-\sigma,-\rho^{\prime},-\rho+\alpha,-\eta+\alpha} f\right)(x),
$$

where $\alpha=[\Re(\eta)]+1$ and $[\Re(\eta)]$ symbolizes the integer part in regard to $\Re(\eta)$. If $\max \{|x|,|y|\}<1$, then the third Appell function $F_{3}$ is defined as

$$
F_{3}\left(\sigma, \sigma^{\prime}, \rho, \rho^{\prime} ; \eta ; x ; y\right)=\sum_{i, j=0}^{\infty} \frac{(\sigma)_{i}\left(\sigma^{\prime}\right)_{j}(\rho)_{i}\left(\rho^{\prime}\right)_{j}}{(\eta)_{i+j}} \frac{x^{i} y^{j}}{i ! j !} .
$$

Here, $(\sigma)_{n}$ is the well-known Pochhammer symbol. Recent papers [2, 17, 25] include a comprehensive demonstration related to the MSM operators together with their properties and applications. Saigo [29] introduced the fractional operators involving Gauss hypergeometric function ${ }_{2} F_{1}()$. For $\sigma, \rho, \eta \in \mathbb{C}, x>0$ along with $\Re(\sigma)>0$, the left- and right-hand sided Saigo fractional integral operators are described as

$$
\left(\mathcal{I}_{0+}^{\sigma, \rho, \eta} f\right)(x)=\frac{x^{-\sigma-\rho}}{\Gamma(\sigma)} \int_{0}^{x}(x-y)^{\sigma-1}{ }_{2} F_{1}\left(\sigma+\rho,-\eta ; \sigma ; 1-\frac{y}{x}\right) f(y) d y
$$

and

$$
\left(\mathcal{I}_{-}^{\sigma, \rho, \eta} f\right)(x)=\frac{1}{\Gamma(\sigma)} \int_{x}^{\infty}(y-x)^{\sigma-1} y^{-\sigma-\rho}{ }_{2} F_{1}\left(\sigma+\rho,-\eta ; \sigma ; 1-\frac{x}{y}\right) f(y) d y,
$$

respectively. The left-hand and right-hand sided Saigo differential operators are defined as

$$
\left(\mathcal{D}_{0+}^{\sigma, \rho, \eta} f\right)(x)=\left(\frac{d}{d x}\right)^{[\Re(\sigma)]+1}\left(\mathcal{I}_{0+}^{-\sigma+[\Re(\sigma)]+1,-\rho-[\Re(\sigma)]-1, \sigma+\eta-[\Re(\sigma)]-1} f\right)(x)
$$


and

$$
\left(\mathcal{D}_{-}^{\sigma, \rho, \eta} f\right)(x)=\left(-\frac{d}{d x}\right)^{[\Re(\sigma)]+1}\left(\mathcal{I}_{-}^{-\sigma+[\Re(\sigma)]+1,-\rho-[\Re(\sigma)]-1, \sigma+\eta} f\right)(x)
$$

For $\rho=-\sigma$ and $\rho=0$ in (14)-(17), the Riemann-Liouville and Erdélyi-Kober fractional operators are obtained respectively (for more explanation see [15]). ${ }_{2} F_{1}$ is associated with $F_{3}$ as

$$
F_{3}(\sigma, \gamma-\sigma, \rho, \gamma-\rho ; \gamma ; x ; y)={ }_{2} F_{1}(\sigma, \rho ; \gamma ; x+y-x y) \text {. }
$$

The MSM fractional operators (9)-(12) are connected to Saigo operators (14)-(17) by

$$
\begin{aligned}
& \left(\mathcal{I}_{0+}^{\sigma, 0, \rho, \rho^{\prime}, \eta} f\right)(x)=\left(\mathcal{I}_{0+}^{\eta, \sigma-\eta,-\rho} f\right)(x), \\
& \left(\mathcal{I}_{-}^{\sigma, 0, \rho, \rho^{\prime}, \eta} f\right)(x)=\left(\mathcal{I}_{-}^{\eta, \sigma-\eta,-\rho} f\right)(x),
\end{aligned}
$$

and

$$
\begin{aligned}
& \left(\mathcal{D}_{0+}^{0, \sigma^{\prime}, \rho, \rho^{\prime}, \eta} f\right)(x)=\left(\mathcal{D}_{0+}^{\eta, \sigma^{\prime}-\eta, \rho^{\prime}-\eta} f\right)(x), \\
& \left(\mathcal{D}_{-}^{0, \sigma^{\prime}, \rho, \rho^{\prime}, \eta} f\right)(x)=\left(\mathcal{D}_{-}^{\eta, \sigma^{\prime}-\eta, \rho^{\prime}-\eta} f\right)(x) .
\end{aligned}
$$

The following are well-known results (see [30]) and will be needed in proving the subsequent theorems.

Lemma 1.1 Let $\sigma, \sigma^{\prime}, \rho, \rho^{\prime}, \eta, \lambda \in \mathbb{C}$ and $\Re(\eta)>0$.

(a) If $\Re(\lambda)>\max \left\{0, \mathfrak{R}\left(\sigma^{\prime}-\rho^{\prime}\right), \mathfrak{R}\left(\sigma+\sigma^{\prime}+\rho-\eta\right)\right\}$, then

$$
\begin{aligned}
\left(\mathcal{I}_{0+}^{\sigma, \sigma^{\prime}, \rho, \rho^{\prime}, \eta} t^{\lambda-1}\right)(x)= & x^{-\sigma-\sigma^{\prime}+\eta+\lambda-1} \\
& \times \frac{\Gamma(\lambda) \Gamma\left(-\sigma^{\prime}+\rho^{\prime}+\lambda\right) \Gamma\left(-\sigma-\sigma^{\prime}-\rho+\eta+\lambda\right)}{\Gamma\left(\rho^{\prime}+\lambda\right) \Gamma\left(-\sigma-\sigma^{\prime}+\eta+\lambda\right) \Gamma\left(-\sigma^{\prime}-\rho+\eta+\lambda\right)} .
\end{aligned}
$$

(b) If $\Re(\lambda)>\max \left\{\Re(\rho), \Re\left(-\sigma-\sigma^{\prime}+\eta\right), \mathfrak{R}\left(-\sigma-\rho^{\prime}+\eta\right)\right\}$, then

$$
\begin{aligned}
\left(\mathcal{I}_{-}^{\sigma, \sigma^{\prime}, \rho, \rho^{\prime}, \eta} t^{-\lambda}\right)(x)= & x^{-\sigma-\sigma^{\prime}+\eta-\lambda} \\
& \times \frac{\Gamma(-\rho+\lambda) \Gamma\left(\sigma+\sigma^{\prime}-\eta+\lambda\right) \Gamma\left(\sigma+\rho^{\prime}-\eta+\lambda\right)}{\Gamma(\lambda) \Gamma(\sigma-\rho+\lambda) \Gamma\left(\sigma+\sigma^{\prime}+\rho^{\prime}-\eta+\lambda\right)} .
\end{aligned}
$$

Motivated by the work of Srivastava et al. [32], we have derived the fractional calculus results associated with the incomplete $I$-functions. In Sect. 2, MSM fractional order integrals of left-hand and right-hand type are derived for the incomplete $I$-functions. In Sect. 3, MSM fractional order derivatives of left-hand and right-hand type are derived for the incomplete I-functions. In Sect. 4, Caputo-type MSM fractional order derivatives of left-hand and right-hand type are derived for the incomplete $I$-functions. In Sect. 5, we have derived the special cases of the incomplete $I$-functions. 


\section{Fractional integration of incomplete $l$-functions}

Some fractional integrations pertaining to the incomplete $I$-functions are presented in this part. First, we shall investigate the MSM fractional order integrals of left-hand side type for the incomplete $I$-functions.

Theorem 2.1 Let $\sigma, \sigma^{\prime}, \rho, \rho^{\prime}, \eta, \lambda, c \in \mathbb{C}$ be so that $\Re(\eta), \mu>0$ and $\Re(\lambda)>\max \left\{0, \Re\left(\sigma^{\prime}-\rho^{\prime}\right)\right.$, $\left.\Re\left(\sigma+\sigma^{\prime}+\rho-\eta\right)\right\}$. Thereupon, for $x>0$,

$$
\begin{array}{r}
\left(\mathcal { I } _ { 0 + } ^ { \sigma , \sigma ^ { \prime } , \rho , \rho ^ { \prime } , \eta } z ^ { \lambda - 1 \gamma } I _ { p , q } ^ { m , n } \left[\begin{array}{c}
c z^{\mu} \\
\left.\left(\begin{array}{c}
\left(a_{1}, \sigma_{1} ; A_{1}: y\right),\left(a_{j}, \sigma_{j} ; A_{j}\right)_{2, p} \\
\left(b_{j}, \rho_{j} ; B_{j}\right)_{1, q}
\end{array}\right]\right)(x) \\
=x^{-\sigma-\sigma^{\prime}+\eta+\lambda-1}
\end{array}\right.\right. \\
\times{ }^{\gamma} I_{p+3, q+3}^{m, n+3}\left[\begin{array}{c}
c x^{\mu} \mid \begin{array}{r}
\left(a_{1}, \sigma_{1} ; A_{1}: y\right),(1-\lambda, \mu ; 1),\left(1+\sigma^{\prime}-\rho^{\prime}-\lambda, \mu ; 1\right), \\
\left(b_{j}, \rho_{j} ; B_{j}\right)_{1, q},\left(1-\rho^{\prime}-\lambda, \mu ; 1\right),
\end{array} \\
\left(1+\sigma+\sigma^{\prime}+\rho-\eta-\lambda, \mu ; 1\right),\left(a_{j}, \sigma_{j} ; A_{j}\right)_{2, p} \\
\left(1+\sigma+\sigma^{\prime}-\eta-\lambda, \mu ; 1\right),\left(1+\sigma+\sigma^{\prime}+\rho-\eta-\lambda, \mu ; 1\right)
\end{array}\right],
\end{array}
$$

provided every member in (22) does exist.

Proof The LHS of (22) is given by

$$
\left(\mathcal{I}_{0+}^{\sigma, \sigma^{\prime}, \rho, \rho^{\prime}, \eta}\left(z^{\lambda-1} \frac{1}{2 \pi i} \int_{\$} \phi(s, y) c^{s} z^{\mu s} d s\right)\right)(x),
$$

where $\phi(s, y)$ is given in (3). Interchanging the order of integration in the above equation yields

$$
\frac{1}{2 \pi i} \int_{\$} \phi(s, y) c^{s}\left(\mathcal{I}_{0+}^{\sigma, \sigma^{\prime}, \rho, \rho^{\prime}, \eta} z^{\lambda+\mu s-1}\right)(x) d s
$$

using the results (20) and (3), we get the RHS of (22).

The properties given below are immediate consequences of definitions (1), (2), and (20), and consequently they are stated without proof here.

Theorem 2.2 Let $\sigma, \sigma^{\prime}, \rho, \rho^{\prime}, \eta, \lambda, c \in \mathbb{C}$ be such that $\Re(\eta), \mu>0$ and $\Re(\lambda)>\max \{0$, $\left.\Re\left(\sigma^{\prime}-\rho^{\prime}\right), \Re\left(\sigma+\sigma^{\prime}+\rho-\eta\right)\right\}$. Thereupon, for $x>0$,

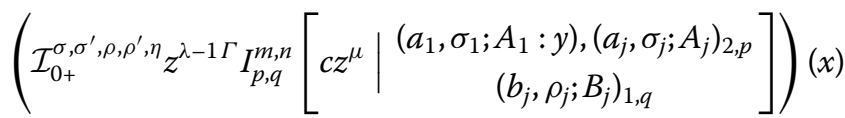

$$
\begin{aligned}
& =x^{-\sigma-\sigma^{\prime}+\eta+\lambda-1} \times \\
& \times{ }^{\Gamma} I_{p+3, q+3}^{m, n+3}\left[c x^{\mu} \mid \begin{array}{c}
\left(a_{1}, \sigma_{1} ; A_{1}: y\right),(1-\lambda, \mu ; 1),\left(1+\sigma^{\prime}-\rho^{\prime}-\lambda, \mu ; 1\right), \\
\left(b_{j}, \rho_{j} ; B_{j}\right)_{1, q},\left(1-\rho^{\prime}-\lambda, \mu ; 1\right),
\end{array}\right. \\
& \left.\begin{array}{c}
\left(1+\sigma+\sigma^{\prime}+\rho-\eta-\lambda, \mu ; 1\right),\left(a_{j}, \sigma_{j} ; A_{j}\right)_{2, p} \\
\left(1+\sigma+\sigma^{\prime}-\eta-\lambda, \mu ; 1\right),\left(1+\sigma+\sigma^{\prime}+\rho-\eta-\lambda, \mu ; 1\right)
\end{array}\right],
\end{aligned}
$$

provided every member in (25) does exist. 
In accordance with (18) and rearranging the involved parameters, we have the subsequent results for Saigo operators.

Corollary 1 Let $\sigma, \rho, \eta, \lambda, c \in \mathbb{C}$ be such that $\Re(\sigma), \mu>0$ and $\Re(\lambda)>\max \{0, \Re(\rho-\eta)\}$. Therefore, for $x>0$,

$$
\begin{array}{r}
\left(\mathcal{I}_{0+}^{\sigma, \rho, \eta} z^{\lambda-1 \gamma} I_{p, q}^{m, n}\left[c z^{\mu} \mid \begin{array}{c}
\left(a_{1}, \sigma_{1} ; A_{1}: y\right),\left(a_{j}, \sigma_{j} ; A_{j}\right)_{2, p} \\
\left(b_{j}, \rho_{j} ; B_{j}\right)_{1, q}
\end{array}\right]\right)(x) \\
=x^{-\rho+\lambda-1 \gamma} I_{p+2, q+2}^{m, n+2}\left[\begin{array}{c}
c x^{\mu} \mid \begin{array}{c}
\left(a_{1}, \sigma_{1} ; A_{1}: y\right),(1-\lambda, \mu ; 1) \\
\left(b_{j}, \rho_{j} ; B_{j}\right)_{1, q},(1+\rho-\lambda, \mu ; 1),
\end{array} \\
(1+\rho-\eta-\lambda, \mu ; 1),\left(a_{j}, \sigma_{j} ; A_{j}\right)_{2, p} \\
(1-\sigma-\eta-\lambda, \mu ; 1)
\end{array}\right],
\end{array}
$$

provided every member in (26) does exist.

Corollary 2 Let $\sigma, \rho, \eta, \lambda, c \in \mathbb{C}$ be such that $\Re(\sigma), \mu>0$ and $\Re(\lambda)>\max \{0, \Re(\rho-\eta)\}$. Thereupon, for $x>0$,

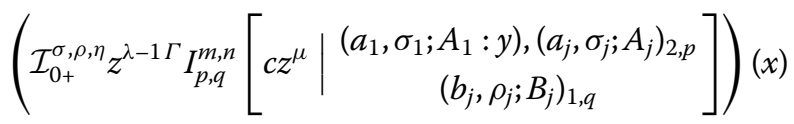

$$
\begin{aligned}
& =x^{-\rho+\lambda-1 \Gamma} I_{p+2, q+2}^{m, n+2}\left[\begin{array}{l|l}
c x^{\mu} & \begin{array}{c}
\left(a_{1}, \sigma_{1} ; A_{1}: y\right),(1-\lambda, \mu ; 1), \\
\left(b_{j}, \rho_{j} ; B_{j}\right)_{1, q},(1+\rho-\lambda, \mu ; 1),
\end{array}
\end{array}\right. \\
& \left.\begin{array}{c}
(1+\rho-\eta-\lambda, \mu ; 1),\left(a_{j}, \sigma_{j} ; A_{j}\right)_{2, p} \\
(1-\sigma-\eta-\lambda, \mu ; 1)
\end{array}\right],
\end{aligned}
$$

provided every member in (27) does exist.

A similar type of image formulas associated with the Riemann-Liouville fractional integral are as follows.

Corollary 3 Let $\sigma, \eta, \lambda, c \in \mathbb{C}$ be such that $\Re(\sigma), \mu>0$ and $\Re(\lambda)>\max \{0, \Re(-\sigma-\eta)\}$. Then, for $x>0$,

$$
\begin{aligned}
& \left(\mathcal{I}_{0+}^{\sigma,-\sigma, \eta} z^{\lambda-1 \gamma} I_{p, q}^{m, n}\left[c z^{\mu} \mid \begin{array}{c}
\left(a_{1}, \sigma_{1} ; A_{1}: y\right),\left(a_{j}, \sigma_{j} ; A_{j}\right)_{2, p} \\
\left(b_{j}, \rho_{j} ; B_{j}\right)_{1, q}
\end{array}\right]\right)(x) \\
& =x^{\sigma+\lambda-1 \gamma} I_{p+1, q+1}^{m, n+1}\left[\begin{array}{c|c}
c x^{\mu} & \begin{array}{c}
\left(a_{1}, \sigma_{1} ; A_{1}: y\right),(1-\lambda, \mu ; 1),\left(a_{j}, \sigma_{j} ; A_{j}\right)_{2, p} \\
\left(b_{j}, \rho_{j} ; B_{j}\right)_{1, q},(1-\sigma-\lambda, \mu ; 1)
\end{array}
\end{array}\right] \text {, }
\end{aligned}
$$

provided every member in (28) does exist. 
Corollary 4 Let $\sigma, \eta, \lambda, c \in \mathbb{C}$ be such that $\Re(\sigma), \mu>0$ and $\Re(\lambda)>\max \{0, \Re(-\sigma-\eta)\}$. Then, for $x>0$,

$$
\begin{aligned}
& \left.\left(\begin{array}{c}
\mathcal{I}_{0+}^{\sigma,-\sigma, \eta} z^{\lambda-1 \Gamma} I_{p, q}^{m, n}\left[c z^{\mu}\right. \\
\left(a_{1}, \sigma_{1} ; A_{1}: y\right),\left(a_{j}, \sigma_{j} ; A_{j}\right)_{2, p} \\
\left(b_{j}, \rho_{j} ; B_{j}\right)_{1, q}
\end{array}\right]\right)(x) \\
& =x^{\sigma+\lambda-1 \Gamma} I_{p+1, q+1}^{m, n+1}\left[c x^{\mu}\right. \\
& \left.\begin{array}{c}
\left(a_{1}, \sigma_{1} ; A_{1}: y\right),(1-\lambda, \mu ; 1),\left(a_{j}, \sigma_{j} ; A_{j}\right)_{2, p} \\
\left(b_{j}, \rho_{j} ; B_{j}\right)_{1, q},(1-\sigma-\lambda, \mu ; 1)
\end{array}\right],
\end{aligned}
$$

provided every member in (29) does exist.

The corresponding corollary (1) introduces the Erdélyi-Kober fractional integral as follows.

Corollary 5 Let $\sigma, \eta, \lambda, c \in \mathbb{C}$ be such that $\Re(\sigma), \mu>0$ and $\Re(\lambda)>\max \{0, \Re(-\eta)\}$. Then, for $x>0$,

$$
\begin{aligned}
& \left(\mathcal{I}_{\eta, \sigma}^{+} z^{\lambda-1 \gamma} I_{p, q}^{m, n}\left[c z^{\mu} \mid \begin{array}{c}
\left(a_{1}, \sigma_{1} ; A_{1}: y\right),\left(a_{j}, \sigma_{j} ; A_{j}\right)_{2, p} \\
\left(b_{j}, \rho_{j} ; B_{j}\right)_{1, q}
\end{array}\right]\right)(x) \\
& =x^{\lambda-1 \gamma} I_{p+1, q+1}^{m, n+1}\left[\begin{array}{c|c}
c x^{\mu} & \begin{array}{c}
\left(a_{1}, \sigma_{1} ; A_{1}: y\right),(1-\eta-\lambda, \mu ; 1),\left(a_{j}, \sigma_{j} ; A_{j}\right)_{2, p} \\
\left(b_{j}, \rho_{j} ; B_{j}\right)_{1, q},(1-\sigma-\eta-\lambda, \mu ; 1)
\end{array}
\end{array}\right],
\end{aligned}
$$

provided every member in (30) does exist.

Corollary 6 Let $\sigma, \eta, \lambda, c \in \mathbb{C}$ be such that $\Re(\sigma), \mu>0$ and $\Re(\lambda)>\max \{0, \Re(-\eta)\}$. Then, for $x>0$,

$$
\begin{aligned}
& \left.\left(\begin{array}{c}
\mathcal{I}_{\eta, \sigma}^{+} z^{\lambda-1 \Gamma} I_{p, q}^{m, n} \\
c z^{\mu}
\end{array} \begin{array}{c}
\left(a_{1}, \sigma_{1} ; A_{1}: y\right),\left(a_{j}, \sigma_{j} ; A_{j}\right)_{2, p} \\
\left(b_{j}, \rho_{j} ; B_{j}\right)_{1, q}
\end{array}\right]\right)(x) \\
& =x^{\lambda-1 \Gamma} I_{p+1, q+1}^{m, n+1}\left[\begin{array}{c|c}
c x^{\mu} & \begin{array}{c}
\left(a_{1}, \sigma_{1} ; A_{1}: y\right),(1-\eta-\lambda, \mu ; 1),\left(a_{j}, \sigma_{j} ; A_{j}\right)_{2, p} \\
\left(b_{j}, \rho_{j} ; B_{j}\right)_{1, q},(1-\sigma-\eta-\lambda, \mu ; 1)
\end{array}
\end{array}\right],
\end{aligned}
$$

provided every member in (31) does exist.

The coming results lead to the right-hand sided MSM fractional order integrals of the incomplete $I$-functions.

Theorem 2.3 Let $\sigma, \sigma^{\prime}, \rho, \rho^{\prime}, \eta, \lambda, c \in \mathbb{C}$ be such that $\Re(\eta), \mu>0$ and $\Re(\lambda)>\max \{\Re(\rho)$, $\left.\Re\left(-\sigma-\sigma^{\prime}+\eta\right), \Re\left(-\sigma-\rho^{\prime}+\eta\right)\right\}$. Then, for $x>0$,

$$
\begin{aligned}
& \left(\mathcal{I}_{-}^{\sigma, \sigma^{\prime}, \rho, \rho^{\prime}, \eta} z^{-\lambda \gamma} I_{p, q}^{m, n}\left[c z^{-\mu} \mid \begin{array}{c}
\left(a_{1}, \sigma_{1} ; A_{1}: y\right),\left(a_{j}, \sigma_{j} ; A_{j}\right)_{2, p} \\
\left(b_{j}, \rho_{j} ; B_{j}\right)_{1, q}
\end{array}\right]\right)(x) \\
& =x^{-\sigma-\sigma^{\prime}+\eta-\lambda} \\
& \times{ }^{\gamma} I_{p+3, q+3}^{m, n+3}\left[c x^{-\mu} \mid \begin{array}{c}
\left(a_{1}, \sigma_{1} ; A_{1}: y\right),(1+\rho-\lambda, \mu ; 1),\left(1-\sigma-\sigma^{\prime}+\eta-\lambda, \mu ; 1\right), \\
\left(b_{j}, \rho_{j} ; B_{j}\right)_{1, q},(1-\lambda, \mu ; 1),
\end{array}\right. \\
& \left.\begin{array}{c}
\left(1-\sigma-\rho^{\prime}+\eta-\lambda, \mu ; 1\right),\left(a_{j}, \sigma_{j} ; A_{j}\right)_{2, p} \\
(1-\sigma+\rho-\lambda, \mu ; 1),\left(1-\sigma-\sigma^{\prime}-\rho^{\prime}+\eta-\lambda, \mu ; 1\right)
\end{array}\right],
\end{aligned}
$$


provided every member in (32) does exist.

Proof The LHS of (32) is given by

$$
\left(\mathcal{I}_{-}^{\sigma, \sigma^{\prime}, \rho, \rho^{\prime}, \eta}\left(z^{-\lambda} \frac{1}{2 \pi i} \int_{\$} \phi(s, y) c^{s} z^{-\mu s} d s\right)\right)(x)
$$

where $\phi(s, y)$ is given in (3). Interchanging the order of integration in the above equation yields

$$
\frac{1}{2 \pi i} \int_{\phi} \phi(s, y) c^{s}\left(\mathcal{I}_{-}^{\sigma, \sigma^{\prime}, \rho, \rho^{\prime}, \eta} z^{-(\lambda+\mu s)}\right)(x) d s,
$$

using the results (21) and (3), we get the RHS of (32).

Theorem 2.4 Let $\sigma, \sigma^{\prime}, \rho, \rho^{\prime}, \eta, \lambda, c \in \mathbb{C}$ be such that $\mathfrak{R}(\eta), \mu>0$ and $\mathfrak{R}(\lambda)>\max \{\Re(\rho)$, $\left.\Re\left(-\sigma-\sigma^{\prime}+\eta\right), \mathfrak{R}\left(-\sigma-\rho^{\prime}+\eta\right)\right\}$. Then, for $x>0$,

$$
\begin{aligned}
& \left(\mathcal{I}_{-}^{\sigma, \sigma^{\prime}, \rho, \rho^{\prime}, \eta} z^{-\lambda \Gamma} I_{p, q}^{m, n}\left[c z^{-\mu} \mid \begin{array}{c}
\left(a_{1}, \sigma_{1} ; A_{1}: y\right),\left(a_{j}, \sigma_{j} ; A_{j}\right)_{2, p} \\
\left(b_{j}, \rho_{j} ; B_{j}\right)_{1, q}
\end{array}\right]\right)(x) \\
& =x^{-\sigma-\sigma^{\prime}+\eta-\lambda} \\
& \times{ }^{\Gamma} I_{p+3, q+3}^{m, n+3}\left[c x^{-\mu} \mid \begin{array}{c}
\left(a_{1}, \sigma_{1} ; A_{1}: y\right),(1+\rho-\lambda, \mu ; 1),\left(1-\sigma-\sigma^{\prime}+\eta-\lambda, \mu ; 1\right) \\
\left(b_{j}, \rho_{j} ; B_{j}\right)_{1, q},(1-\lambda, \mu ; 1),
\end{array}\right. \\
& \left.\begin{array}{c}
\left(1-\sigma-\rho^{\prime}+\eta-\lambda, \mu ; 1\right),\left(a_{j}, \sigma_{j} ; A_{j}\right)_{2, p} \\
(1-\sigma+\rho-\lambda, \mu ; 1),\left(1-\sigma-\sigma^{\prime}-\rho^{\prime}+\eta-\lambda, \mu ; 1\right)
\end{array}\right],
\end{aligned}
$$

provided every member in (35) does exist.

The Saigo, Riemann-Liouville, and Erdélyi-Kober fractional order integrals of the incomplete $I$-functions are given below.

Corollary 7 Let $\sigma, \rho, \eta, \lambda, c \in \mathbb{C}$ be such that $\mathfrak{R}(\sigma), \mu>0$ and $\Re(\lambda)>\max \{\Re(-\rho), \Re(-\eta)\}$. Then, for $x>0$,

$$
\begin{aligned}
& \left(\mathcal{I}_{-}^{\sigma, \rho, \eta} z^{-\lambda \gamma} I_{p, q}^{m, n}\left[c z^{-\mu} \mid \begin{array}{c}
\left(a_{1}, \sigma_{1} ; A_{1}: y\right),\left(a_{j}, \sigma_{j} ; A_{j}\right)_{2, p} \\
\left(b_{j}, \rho_{j} ; B_{j}\right)_{1, q}
\end{array}\right]\right)(x)
\end{aligned}
$$

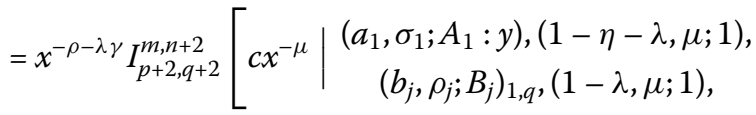

$$
\begin{aligned}
& \left.\begin{array}{c}
(1-\rho-\lambda, \mu ; 1),\left(a_{j}, \sigma_{j} ; A_{j}\right)_{2, p} \\
(1-\sigma-\rho-\eta-\lambda, \mu ; 1)
\end{array}\right]
\end{aligned}
$$

provided every member in (36) does exist. 
Corollary 8 Let $\sigma, \rho, \eta, \lambda, c \in \mathbb{C}$ be such that $\mathfrak{R}(\sigma), \mu>0$ and $\mathfrak{R}(\lambda)>\max \{\Re(-\rho), \mathfrak{R}(-\eta)\}$. Then, for $x>0$,

$$
\begin{aligned}
& \left(\mathcal{I}_{-}^{\sigma, \rho, \eta} z^{-\lambda \Gamma} I_{p, q}^{m, n}\left[\begin{array}{c|c}
c z^{-\mu} & \left(a_{1}, \sigma_{1} ; A_{1}: y\right),\left(a_{j}, \sigma_{j} ; A_{j}\right)_{2, p} \\
\left(b_{j}, \rho_{j} ; B_{j}\right)_{1, q}
\end{array}\right]\right)(x)
\end{aligned}
$$

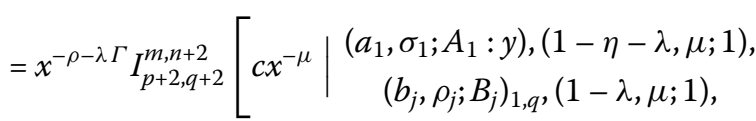

$$
\begin{aligned}
& \left.\begin{array}{c}
(1-\rho-\lambda, \mu ; 1),\left(a_{j}, \sigma_{j} ; A_{j}\right)_{2, p} \\
(1-\sigma-\rho-\eta-\lambda, \mu ; 1)
\end{array}\right],
\end{aligned}
$$

provided every member in (37) does exist.

Corollary 9 Let $\sigma, \eta, \lambda, c \in \mathbb{C}$ be such that $\Re(\sigma), \mu>0$ and $\Re(\lambda)>\max \{\Re(\sigma), \Re(-\eta)\}$. Then, for $x>0$,

$$
\left.\begin{array}{l}
\left(\mathcal { I } _ { - } ^ { \sigma , - \sigma , \eta } z ^ { - \lambda \gamma } I _ { p , q } ^ { m , n } \left[c z^{-\mu}\right.\right. \\
\left.\left.\begin{array}{c}
\left(a_{1}, \sigma_{1} ; A_{1}: y\right),\left(a_{j}, \sigma_{j} ; A_{j}\right)_{2, p} \\
\left(b_{j}, \rho_{j} ; B_{j}\right)_{1, q}
\end{array}\right]\right)(x)
\end{array}\right)
$$

provided every member in (38) does exist.

Corollary 10 Let $\sigma, \eta, \lambda, c \in \mathbb{C}$ be such that $\Re(\sigma), \mu>0$ and $\Re(\lambda)>\max \{\Re(\sigma), \Re(-\eta)\}$. Then, for $x>0$,

$$
\left.\begin{array}{l}
\left(\mathcal { I } _ { - } ^ { \sigma , - \sigma , \eta } z ^ { - \lambda \Gamma } I _ { p , q } ^ { m , n } \left[c z^{-\mu}\right.\right. \\
\left.\left.\begin{array}{c}
\left(a_{1}, \sigma_{1} ; A_{1}: y\right),\left(a_{j}, \sigma_{j} ; A_{j}\right)_{2, p} \\
\left(b_{j}, \rho_{j} ; B_{j}\right)_{1, q}
\end{array}\right]\right)(x)
\end{array}\right]
$$

provided every member in (39) does exist.

Corollary 11 Let $\sigma, \eta, \lambda, c \in \mathbb{C}$ be such that $\Re(\sigma), \mu>0$ and $\Re(\lambda)>\max \{0, \Re(-\eta)\}$. Then the right-hand Erdélyi-Kober integration $\mathcal{K}_{\eta, \sigma}^{-}\left(=\mathcal{I}_{-}^{\sigma, 0, \eta}\right)$ for $x>0$,

$$
\begin{aligned}
& \left(\mathcal{K}_{\eta, \sigma}^{-} z^{-\lambda \gamma} I_{p, q}^{m, n}\left[c z^{-\mu} \mid \begin{array}{c}
\left(a_{1}, \sigma_{1} ; A_{1}: y\right),\left(a_{j}, \sigma_{j} ; A_{j}\right)_{2, p} \\
\left(b_{j}, \rho_{j} ; B_{j}\right)_{1, q}
\end{array}\right]\right)(x)
\end{aligned}
$$

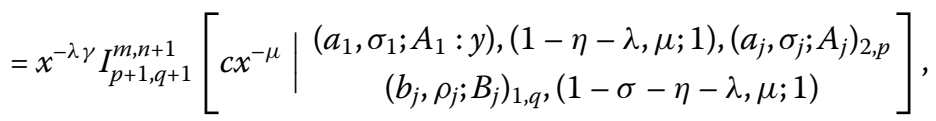

provided every member in (40) does exist. 
Corollary 12 Let $\sigma, \eta, \lambda, c \in \mathbb{C}$ be such that $\Re(\sigma), \mu>0$ and $\Re(\lambda)>\max \{0, \Re(-\eta)\}$. Then the right-hand Erdélyi-Kober integration $\mathcal{K}_{\eta, \sigma}^{-}\left(=\mathcal{I}_{-}^{\sigma, 0, \eta}\right)$ for $x>0$ is as follows:

$$
\begin{aligned}
& \left(\mathcal{K}_{\eta, \sigma}^{-} z^{-\lambda \Gamma} I_{p, q}^{m, n}\left[c z^{-\mu} \mid \begin{array}{c}
\left(a_{1}, \sigma_{1} ; A_{1}: y\right),\left(a_{j}, \sigma_{j} ; A_{j}\right)_{2, p} \\
\left(b_{j}, \rho_{j} ; B_{j}\right)_{1, q}
\end{array}\right]\right)(x) \\
& =x^{-\lambda \Gamma} I_{p+1, q+1}^{m, n+1}\left[\begin{array}{c|c}
c x^{-\mu} & \begin{array}{c}
\left(a_{1}, \sigma_{1} ; A_{1}: y\right),(1-\eta-\lambda, \mu ; 1),\left(a_{j}, \sigma_{j} ; A_{j}\right)_{2, p} \\
\left(b_{j}, \rho_{j} ; B_{j}\right)_{1, q},(1-\sigma-\eta-\lambda, \mu ; 1)
\end{array}
\end{array}\right],
\end{aligned}
$$

provided every member in (41) does exist.

\section{Fractional differentiation of incomplete $l$-functions}

Right now, we study the MSM fractional order derivatives pertaining to the incomplete $I$ functions. The following are well-known results and will be used in subsequent theorems (see [30]).

Lemma 3.1 Let $\sigma, \sigma^{\prime}, \rho, \rho^{\prime}, \eta, \lambda \in \mathbb{C}$.

(a) If $\Re(\lambda)>\max \left\{0, \Re(-\sigma+\rho), \mathfrak{R}\left(-\sigma-\sigma^{\prime}-\rho^{\prime}+\eta\right)\right\}$, then

$$
\begin{aligned}
\left(\mathcal{D}_{0+}^{\sigma, \sigma^{\prime}, \rho, \rho^{\prime}, \eta} t^{\lambda-1}\right)(x)= & x^{\sigma+\sigma^{\prime}-\eta+\lambda-1} \\
& \times \frac{\Gamma(\lambda) \Gamma(\sigma-\rho+\lambda) \Gamma\left(\sigma+\sigma^{\prime}+\rho^{\prime}-\eta+\lambda\right)}{\Gamma(-\rho+\lambda) \Gamma\left(\sigma+\sigma^{\prime}-\eta+\lambda\right) \Gamma\left(\sigma+\rho^{\prime}-\eta+\lambda\right)} .
\end{aligned}
$$

(b) If $\Re(\lambda)>\max \left\{\Re\left(-\rho^{\prime}\right), \Re\left(\sigma^{\prime}+\rho-\eta\right), \Re\left(\sigma+\sigma^{\prime}-\eta\right)+[\Re(\eta)]+1\right\}$, then

$$
\begin{aligned}
\left(\mathcal{D}_{-}^{\sigma, \sigma^{\prime}, \rho, \rho^{\prime}, \eta} t^{-\lambda}\right)(x)= & x^{\sigma+\sigma^{\prime}-\eta-\lambda} \\
& \times \frac{\Gamma\left(\rho^{\prime}+\lambda\right) \Gamma\left(-\sigma-\sigma^{\prime}+\eta+\lambda\right) \Gamma\left(-\sigma^{\prime}-\rho+\eta+\lambda\right)}{\Gamma(\lambda) \Gamma\left(-\sigma^{\prime}+\rho^{\prime}+\lambda\right) \Gamma\left(-\sigma-\sigma^{\prime}-\rho+\eta+\lambda\right)} .
\end{aligned}
$$

Now, we represent the left-hand sided MSM fractional derivatives of the incomplete $I$-functions.

Theorem 3.2 Let $\sigma, \sigma^{\prime}, \rho, \rho^{\prime}, \eta, \lambda, c \in \mathbb{C}$ be such that $\mu>0$ and $\Re(\lambda)>\max \{0, \Re(-\sigma+\rho)$, $\left.\Re\left(-\sigma-\sigma^{\prime}-\rho^{\prime}+\eta\right)\right\}$. Then, for $x>0$,

$$
\begin{aligned}
& \left(\mathcal{D}_{0+}^{\sigma, \sigma^{\prime}, \rho, \rho^{\prime}, \eta} z^{\lambda-1 \gamma} I_{p, q}^{m, n}\left[c z^{\mu} \mid \begin{array}{c}
\left(a_{1}, \sigma_{1} ; A_{1}: y\right),\left(a_{j}, \sigma_{j} ; A_{j}\right)_{2, p} \\
\left(b_{j}, \rho_{j} ; B_{j}\right)_{1, q}
\end{array}\right]\right)(x) \\
& =x^{\sigma+\sigma^{\prime}-\eta+\lambda-1} \\
& \times{ }^{\gamma} I_{p+3, q+3}^{m, n+3}\left[c x^{\mu} \mid \begin{array}{c}
\left(a_{1}, \sigma_{1} ; A_{1}: y\right),(1-\lambda, \mu ; 1),(1-\sigma+\rho-\lambda, \mu ; 1), \\
\left(b_{j}, \rho_{j} ; B_{j}\right)_{1, q},(1+\rho-\lambda, \mu ; 1),
\end{array}\right. \\
& \left.\begin{array}{c}
\left(1-\sigma-\sigma^{\prime}-\rho^{\prime}+\eta-\lambda, \mu ; 1\right),\left(a_{j}, \sigma_{j} ; A_{j}\right)_{2, p} \\
\left(1-\sigma-\sigma^{\prime}+\eta-\lambda, \mu ; 1\right),\left(1-\sigma-\rho^{\prime}+\eta-\lambda, \mu ; 1\right)
\end{array}\right],
\end{aligned}
$$

provided every member in (44) does exist. 
Proof The LHS of (44) is given by

$$
\left(\mathcal{D}_{0+}^{\sigma, \sigma^{\prime}, \rho, \rho^{\prime}, \eta}\left(z^{\lambda-1} \frac{1}{2 \pi i} \int_{\$} \phi(s, y) c^{s} z^{\mu s} d s\right)\right)(x),
$$

where $\phi(s, y)$ is given in (3). Interchanging the order of integration in the above equation yields

$$
\frac{1}{2 \pi i} \int_{\$} \phi(s, y) c^{s}\left(\mathcal{D}_{0+}^{\sigma, \sigma^{\prime}, \rho, \rho^{\prime}, \eta} z^{\lambda+\mu s-1}\right)(x) d s,
$$

using the results (42) and (3), we get the RHS of (44).

The properties given below are immediate consequences of definitions (1), (2), and (42), and consequently they are disposed without demonstration as follows.

Theorem 3.3 Let $\sigma, \sigma^{\prime}, \rho, \rho^{\prime}, \eta, \lambda, c \in \mathbb{C}$ be such that $\mu>0$ and $\Re(\lambda)>\max \{0, \Re(-\sigma+\rho)$, $\left.\Re\left(-\sigma-\sigma^{\prime}-\rho^{\prime}+\eta\right)\right\}$. Then, for $x>0$,

$$
\begin{aligned}
& \left(\mathcal{D}_{0+}^{\sigma, \sigma^{\prime}, \rho, \rho^{\prime}, \eta} z^{\lambda-1 \Gamma} I_{p, q}^{m, n}\left[c z^{\mu} \mid \begin{array}{c}
\left(a_{1}, \sigma_{1} ; A_{1}: y\right),\left(a_{j}, \sigma_{j} ; A_{j}\right)_{2, p} \\
\left(b_{j}, \rho_{j} ; B_{j}\right)_{1, q}
\end{array}\right]\right)(x) \\
& =x^{\sigma+\sigma^{\prime}-\eta+\lambda-1} \\
& \times{ }^{\Gamma} I_{p+3, q+3}^{m, n+3}\left[c x^{\mu} \mid \begin{array}{c}
\left(a_{1}, \sigma_{1} ; A_{1}: y\right),(1-\lambda, \mu ; 1),(1-\sigma+\rho-\lambda, \mu ; 1), \\
\left(b_{j}, \rho_{j} ; B_{j}\right)_{1, q},(1+\rho-\lambda, \mu ; 1),
\end{array}\right. \\
& \left.\begin{array}{c}
\left(1-\sigma-\sigma^{\prime}-\rho^{\prime}+\eta-\lambda, \mu ; 1\right),\left(a_{j}, \sigma_{j} ; A_{j}\right)_{2, p} \\
\left(1-\sigma-\sigma^{\prime}+\eta-\lambda, \mu ; 1\right),\left(1-\sigma-\rho^{\prime}+\eta-\lambda, \mu ; 1\right)
\end{array}\right],
\end{aligned}
$$

provided every member in (47) does exist.

The coming image formulas for incomplete $I$-functions involving the Saigo, RiemannLiouville, and Erdélyi-Kober fractional derivatives are as follows.

Corollary 13 Let $\sigma, \rho, \eta, \lambda, c \in \mathbb{C}$ be such that $\mu>0$ and $\Re(\lambda)>\max \{0, \Re(-\sigma-\rho-\eta)\}$. Then, for $x>0$,

$$
\begin{array}{r}
\left(\mathcal{D}_{0+}^{\sigma, \rho, \eta} z^{\lambda-1 \gamma} I_{p, q}^{m, n}\left[c z^{\mu} \mid \begin{array}{c}
\left(a_{1}, \sigma_{1} ; A_{1}: y\right),\left(a_{j}, \sigma_{j} ; A_{j}\right)_{2, p} \\
\left(b_{j}, \rho_{j} ; B_{j}\right)_{1, q}
\end{array}\right]\right)(x) \\
=x^{\rho+\lambda-1 \gamma} I_{p+2, q+2}^{m, n+2}\left[\begin{array}{c}
c x^{\mu} \mid \begin{array}{c}
\left(a_{1}, \sigma_{1} ; A_{1}: y\right),(1-\lambda, \mu ; 1), \\
\left(b_{j}, \rho_{j} ; B_{j}\right)_{1, q},(1-\rho-\lambda, \mu ; 1),
\end{array} \\
(1-\sigma-\rho-\eta-\lambda, \mu ; 1),\left(a_{j}, \sigma_{j} ; A_{j}\right)_{2, p} \\
(1-\eta-\lambda, \mu ; 1)
\end{array}\right],
\end{array}
$$

provided every member in (48) does exist. 
Corollary 14 Let $\sigma, \rho, \eta, \lambda, c \in \mathbb{C}$ be such that $\mu>0$ and $\Re(\lambda)>\max \{0, \Re(-\sigma-\rho-\eta)\}$. Then, for $x>0$,

$$
\begin{aligned}
& \left(\mathcal{D}_{0+}^{\sigma, \rho, \eta} z^{\lambda-1 \Gamma} I_{p, q}^{m, n}\left[c z^{\mu} \mid \begin{array}{c}
\left(a_{1}, \sigma_{1} ; A_{1}: y\right),\left(a_{j}, \sigma_{j} ; A_{j}\right)_{2, p} \\
\left(b_{j}, \rho_{j} ; B_{j}\right)_{1, q}
\end{array}\right]\right)(x) \\
& =x^{\rho+\lambda-1 \Gamma} I_{p+2, q+2}^{m, n+2}\left[c x^{\mu} \mid \begin{array}{c}
\left(a_{1}, \sigma_{1} ; A_{1}: y\right),(1-\lambda, \mu ; 1), \\
\left(b_{j}, \rho_{j} ; B_{j}\right)_{1, q},(1-\rho-\lambda, \mu ; 1),
\end{array}\right. \\
& \left.\begin{array}{c}
(1-\sigma-\rho-\eta-\lambda, \mu ; 1),\left(a_{j}, \sigma_{j} ; A_{j}\right)_{2, p} \\
(1-\eta-\lambda, \mu ; 1)
\end{array}\right],
\end{aligned}
$$

provided every member in (49) does exist.

Corollary 15 Let $\sigma, \eta, \lambda, c \in \mathbb{C}$ be such that $\mu>0$ and $\Re(\lambda)>\max \{0, \Re(-\eta)\}$. Then, for $x>0$,

$$
\begin{aligned}
& \left(\mathcal{D}_{0+}^{\sigma,-\sigma, \eta} z^{\lambda-1 \gamma} I_{p, q}^{m, n}\left[c z^{\mu} \mid \begin{array}{c}
\left(a_{1}, \sigma_{1} ; A_{1}: y\right),\left(a_{j}, \sigma_{j} ; A_{j}\right)_{2, p} \\
\left(b_{j}, \rho_{j} ; B_{j}\right)_{1, q}
\end{array}\right]\right)(x)
\end{aligned}
$$

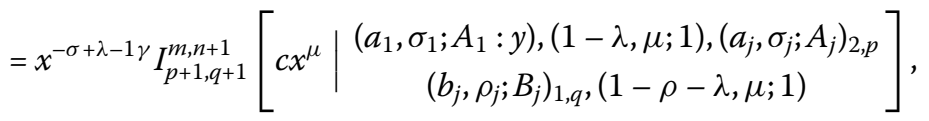

provided every member in (50) does exist.

Corollary 16 Let $\sigma, \eta, \lambda, c \in \mathbb{C}$ be such that $\mu>0$ and $\Re(\lambda)>\max \{0, \Re(-\eta)\}$. Then, for $x>0$,

$$
\begin{gathered}
\left.\left(\begin{array}{c}
\mathcal{D}_{0+}^{\sigma,-\sigma, \eta} z^{\lambda-1} \Gamma I_{p, q}^{m, n}\left[c z^{\mu}\right. \\
\left(a_{1}, \sigma_{1} ; A_{1}: y\right),\left(a_{j}, \sigma_{j} ; A_{j}\right)_{2, p} \\
\left(b_{j}, \rho_{j} ; B_{j}\right)_{1, q}
\end{array}\right]\right)(x) \\
=x^{-\sigma+\lambda-1 \Gamma} I_{p+1, q+1}^{m, n+1}\left[c x^{\mu} \mid \begin{array}{c}
\left(a_{1}, \sigma_{1} ; A_{1}: y\right),(1-\lambda, \mu ; 1),\left(a_{j}, \sigma_{j} ; A_{j}\right)_{2, p} \\
\left(b_{j}, \rho_{j} ; B_{j}\right)_{1, q},(1-\rho-\lambda, \mu ; 1)
\end{array}\right],
\end{gathered}
$$

provided every member in (51) does exist.

Corollary 17 Let $\sigma, \eta, \lambda, c \in \mathbb{C}$ be such that $\mu>0$ and $\Re(\lambda)>\max \{0, \Re(-\sigma-\eta)\}$. Then the left-hand sided Erdélyi-Kober differential $\mathcal{D}_{\eta, \sigma}^{+}\left(=\mathcal{D}_{0_{+}}^{\sigma, 0, \eta}\right)$ of the incomplete I-function is given for $x>0$ by

$$
\begin{aligned}
& \left.\left(\begin{array}{c}
\mathcal{D}_{\eta, \sigma}^{+} z^{\lambda-1 \gamma} I_{p, q}^{m, n}\left[c z^{\mu}\right. \\
=x^{\lambda-1} \times \\
\left.\left(a_{1}, \sigma_{1} ; A_{1}: y\right),\left(a_{j}, \sigma_{j} ; A_{j}\right)_{1, q}\right)_{2, p}
\end{array}\right]\right)(x) \\
& \quad \times{ }^{\gamma} I_{p+1, q+1}^{m, n+1}\left[c x^{\mu}\right. \\
& \left.\begin{array}{c}
\left(a_{1}, \sigma_{1} ; A_{1}: y\right),(1-\sigma-\eta-\lambda, \mu ; 1),\left(a_{j}, \sigma_{j} ; A_{j}\right)_{2, p} \\
\left(b_{j}, \rho_{j} ; B_{j}\right)_{1, q},(1-\eta-\lambda, \mu ; 1)
\end{array}\right],
\end{aligned}
$$

provided every member in (52) does exist. 
Corollary 18 Let $\sigma, \eta, \lambda, c \in \mathbb{C}$ be such that $\mu>0$ and $\Re(\lambda)>\max \{0, \Re(-\sigma-\eta)\}$. Then the left-hand sided Erdélyi-Kober differential $\mathcal{D}_{\eta, \sigma}^{+}\left(=\mathcal{D}_{0+}^{\sigma, 0, \eta}\right)$ of the incomplete I-function is given for $x>0$ by

$$
\begin{aligned}
& \left(\mathcal{D}_{\eta, \sigma}^{+} z^{\lambda-1 \Gamma} I_{p, q}^{m, n}\left[c z^{\mu} \mid \begin{array}{c}
\left(a_{1}, \sigma_{1} ; A_{1}: y\right),\left(a_{j}, \sigma_{j} ; A_{j}\right)_{2, p} \\
\left(b_{j}, \rho_{j} ; B_{j}\right)_{1, q}
\end{array}\right]\right)(x) \\
& =x^{\lambda-1} \\
& \times{ }^{\Gamma} I_{p+1, q+1}^{m, n+1}\left[c x^{\mu} \mid \begin{array}{c}
\left(a_{1}, \sigma_{1} ; A_{1}: y\right),(1-\sigma-\eta-\lambda, \mu ; 1),\left(a_{j}, \sigma_{j} ; A_{j}\right)_{2, p} \\
\left(b_{j}, \rho_{j} ; B_{j}\right)_{1, q},(1-\eta-\lambda, \mu ; 1)
\end{array}\right],
\end{aligned}
$$

provided every member in (53) does exist.

The next result leads to the right-hand MSM fractional derivative related to the incomplete $I$-functions.

Theorem 3.4 Let $\sigma, \sigma^{\prime}, \rho, \rho^{\prime}, \eta, \lambda, c \in \mathbb{C}$ be such that $\mu>0$ and $\Re(\lambda)>\max \left\{\Re\left(-\rho^{\prime}\right)\right.$, $\left.\Re\left(\sigma^{\prime}+\rho-\eta\right), \Re\left(\sigma+\sigma^{\prime}-\eta\right)+[\Re(\eta)]+1\right\}$. Then, for $x>0$,

$$
\begin{aligned}
& \left(\mathcal{D}_{-}^{\sigma, \sigma^{\prime}, \rho, \rho^{\prime}, \eta} z^{-\lambda \gamma} I_{p, q}^{m, n}\left[c z^{-\mu} \mid \begin{array}{c}
\left(a_{1}, \sigma_{1} ; A_{1}: y\right),\left(a_{j}, \sigma_{j} ; A_{j}\right)_{2, p} \\
\left(b_{j}, \rho_{j} ; B_{j}\right)_{1, q}
\end{array}\right]\right)(x) \\
& =x^{\sigma+\sigma^{\prime}-\eta-\lambda} \\
& \times{ }^{\gamma} I_{p+3, q+3}^{m, n+3}\left[c x^{-\mu} \mid \begin{array}{c}
\left(a_{1}, \sigma_{1} ; A_{1}: y\right),\left(1-\rho^{\prime}-\lambda, \mu ; 1\right),\left(1+\sigma+\sigma^{\prime}-\eta-\lambda, \mu ; 1\right), \\
\left(b_{j}, \rho_{j} ; B_{j}\right)_{1, q},(1-\lambda, \mu ; 1),
\end{array}\right. \\
& \left.\begin{array}{c}
\left(1+\sigma^{\prime}+\rho-\eta-\lambda, \mu ; 1\right),\left(a_{j}, \sigma_{j} ; A_{j}\right)_{2, p} \\
\left(1+\sigma^{\prime}-\rho^{\prime}-\lambda, \mu ; 1\right),\left(1+\sigma+\sigma^{\prime}+\rho-\eta-\lambda, \mu ; 1\right)
\end{array}\right],
\end{aligned}
$$

provided every member in (54) does exist.

Proof The LHS of (54) is given by

$$
\left(\mathcal{D}_{-}^{\sigma, \sigma^{\prime}, \rho, \rho^{\prime}, \eta}\left(z^{-\lambda} \frac{1}{2 \pi i} \int_{\$} \phi(s, y) c^{s} z^{-\mu s} d s\right)\right)(x)
$$

where $\phi(s, y)$ is given in (3). Interchanging the order of integration in the above equation yields

$$
\frac{1}{2 \pi i} \int_{\$} \phi(s, y) c^{s}\left(\mathcal{D}_{-}^{\sigma, \sigma^{\prime}, \rho, \rho^{\prime}, \eta} z^{-(\lambda+\mu s)}\right)(x) d s
$$

using the results (43) and (3), we get the RHS of (54). 
Theorem 3.5 Let $\sigma, \sigma^{\prime}, \rho, \rho^{\prime}, \eta, \lambda, c \in \mathbb{C}$ be such that $\mu>0$ and $\Re(\lambda)>\max \left\{\Re\left(-\rho^{\prime}\right)\right.$, $\left.\Re\left(\sigma^{\prime}+\rho-\eta\right), \Re\left(\sigma+\sigma^{\prime}-\eta\right)+[\Re(\eta)]+1\right\}$. Then, for $x>0$,

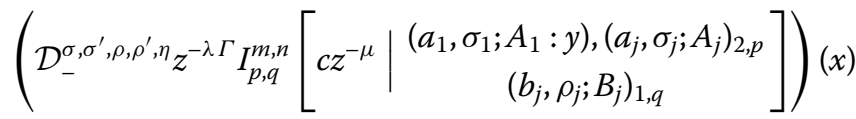

$$
\begin{aligned}
& =x^{\sigma+\sigma^{\prime}-\eta-\lambda} \\
& \times{ }^{\Gamma} I_{p+3, q+3}^{m, n+3}\left[c x^{-\mu} \mid \begin{array}{c}
\left(a_{1}, \sigma_{1} ; A_{1}: y\right),\left(1-\rho^{\prime}-\lambda, \mu ; 1\right),\left(1+\sigma+\sigma^{\prime}-\eta-\lambda, \mu ; 1\right), \\
\left(b_{j}, \rho_{j} ; B_{j}\right)_{1, q},(1-\lambda, \mu ; 1),
\end{array}\right. \\
& \left.\begin{array}{c}
\left(1+\sigma^{\prime}+\rho-\eta-\lambda, \mu ; 1\right),\left(a_{j}, \sigma_{j} ; A_{j}\right)_{2, p} \\
\left(1+\sigma^{\prime}-\rho^{\prime}-\lambda, \mu ; 1\right),\left(1+\sigma+\sigma^{\prime}+\rho-\eta-\lambda, \mu ; 1\right)
\end{array}\right],
\end{aligned}
$$

provided every member in (57) does exist.

The fractional order derivatives of Saigo, Riemann-Liouville, and Erdélyi-Kober type involving the incomplete $I$-functions are given as follows.

Corollary 19 Let $\sigma, \rho, \eta, \lambda, c \in \mathbb{C}$ be such that $\mu>0$ and $\Re(\lambda)>\max \{\Re(-\sigma-\eta)$, $\Re(\rho)+[\Re(\sigma)]+1\}$. Then the right-hand Saigo derivative of the incomplete I-function is given for $x>0$ by

$$
\begin{aligned}
& \left(\mathcal{D}_{-}^{\sigma, \rho, \eta} z^{-\lambda \gamma} I_{p, q}^{m, n}\left[c z^{-\mu} \mid \begin{array}{c}
\left(a_{1}, \sigma_{1} ; A_{1}: y\right),\left(a_{j}, \sigma_{j} ; A_{j}\right)_{2, p} \\
\left(b_{j}, \rho_{j} ; B_{j}\right)_{1, q}
\end{array}\right]\right)(x)
\end{aligned}
$$

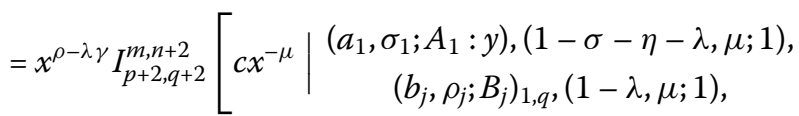

$$
\begin{aligned}
& \left.\begin{array}{c}
(1+\rho-\lambda, \mu ; 1),\left(a_{j}, \sigma_{j} ; A_{j}\right)_{2, p} \\
(1+\rho-\eta-\lambda, \mu ; 1)
\end{array}\right],
\end{aligned}
$$

provided every member in (58) does exist.

Corollary 20 Let $\sigma, \rho, \eta, \lambda, c \in \mathbb{C}$ be such that $\mu>0$ and $\Re(\lambda)>\max \{\Re(-\sigma-\eta)$, $\Re(\rho)+[\Re(\sigma)]+1\}$. Then the right-hand Saigo derivative of the incomplete I-function is given for $x>0$ by

$$
\begin{aligned}
& \left(\mathcal{D}_{-}^{\sigma, \rho, \eta} z^{-\lambda \Gamma} I_{p, q}^{m, n}\left[c z^{-\mu} \mid \begin{array}{c}
\left(a_{1}, \sigma_{1} ; A_{1}: y\right),\left(a_{j}, \sigma_{j} ; A_{j}\right)_{2, p} \\
\left(b_{j}, \rho_{j} ; B_{j}\right)_{1, q}
\end{array}\right]\right)(x)
\end{aligned}
$$

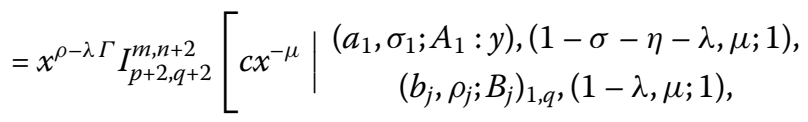

$$
\begin{aligned}
& \left.\begin{array}{c}
(1+\rho-\lambda, \mu ; 1),\left(a_{j}, \sigma_{j} ; A_{j}\right)_{2, p} \\
(1+\rho-\eta-\lambda, \mu ; 1)
\end{array}\right],
\end{aligned}
$$

provided every member in (59) does exist. 
Corollary 21 Let $\sigma, \eta, \lambda, c \in \mathbb{C}$ be such that $\mu>0$ and $\Re(\lambda)>\max \{\Re(-\sigma-\eta), \Re(-\sigma)+$ $[\Re(\sigma)]+1\}$. Then, for $x>0$,

$$
\left.\begin{array}{l}
\left(\mathcal { D } _ { - } ^ { \sigma , - \sigma , \eta } z ^ { - \lambda \gamma } I _ { p , q } ^ { m , n } \left[c z^{-\mu}\right.\right. \\
\left.\left.\begin{array}{c}
\left(a_{1}, \sigma_{1} ; A_{1}: y\right),\left(a_{j}, \sigma_{j} ; A_{j}\right)_{2, p} \\
\left(b_{j}, \rho_{j} ; B_{j}\right)_{1, q}
\end{array}\right]\right)(x)
\end{array}\right)
$$

provided every member in (60) does exist.

Corollary 22 Let $\sigma, \eta, \lambda, c \in \mathbb{C}$ be such that $\mu>0$ and $\Re(\lambda)>\max \{\Re(-\sigma-\eta), \Re(-\sigma)+$ $[\Re(\sigma)]+1\}$. Then, for $x>0$,

$$
\begin{aligned}
& \left(\mathcal { D } _ { - } ^ { \sigma , - \sigma , \eta } z ^ { - \lambda \Gamma } I _ { p , q } ^ { m , n } \left[c z^{-\mu}\right.\right. \\
& \left.\left.\quad \begin{array}{c}
\left(a_{1}, \sigma_{1} ; A_{1}: y\right),\left(a_{j}, \sigma_{j} ; A_{j}\right)_{2, p} \\
\left(b_{j}, \rho_{j} ; B_{j}\right)_{1, q}
\end{array}\right]\right)(x) \\
& =x^{-\sigma-\lambda \Gamma} I_{p+1, q+1}^{m, n+1}\left[c x^{-\mu}\right. \\
& \left.\begin{array}{c}
\left(a_{1}, \sigma_{1} ; A_{1}: y\right),(1+\rho-\lambda, \mu ; 1),\left(a_{j}, \sigma_{j} ; A_{j}\right)_{2, p} \\
\left(b_{j}, \rho_{j} ; B_{j}\right)_{1, q},(1-\lambda, \mu ; 1)
\end{array}\right],
\end{aligned}
$$

provided every member in (61) does exist.

Corollary 23 Let $\sigma, \eta, \lambda, c \in \mathbb{C}$ be such that $\mu>0$ and $\Re(\lambda)>\max \{\Re(-\sigma-\eta),[\Re(\sigma)]+1\}$. Then the right-hand Erdélyi-Kober fractional differentiation $\mathcal{D}_{\eta, \sigma}^{-}\left(=\mathcal{D}_{-}^{\sigma, 0, \eta}\right)$ for $x>0$ is

$$
\begin{aligned}
& \left(\mathcal{D}_{\eta, \sigma}^{-} z^{-\lambda \gamma} I_{p, q}^{m, n}\left[c z^{-\mu} \mid \begin{array}{c}
\left(a_{1}, \sigma_{1} ; A_{1}: y\right),\left(a_{j}, \sigma_{j} ; A_{j}\right)_{2, p} \\
\left(b_{j}, \rho_{j} ; B_{j}\right)_{1, q}
\end{array}\right]\right)(x)
\end{aligned}
$$

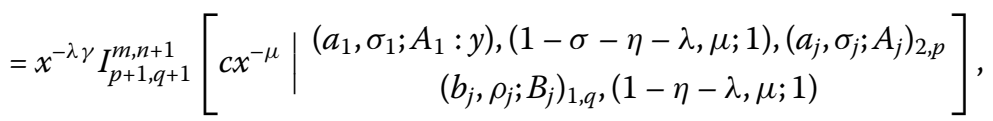

provided every member in (62) does exist.

Corollary 24 Let $\sigma, \eta, \lambda, c \in \mathbb{C}$ be such that $\mu>0$ and $\Re(\lambda)>\max \{\Re(-\sigma-\eta),[\Re(\sigma)]+1\}$. Then the right-hand Erdélyi-Kober fractional differentiation $\mathcal{D}_{\eta, \sigma}^{-}\left(=\mathcal{D}_{-}^{\sigma, 0, \eta}\right)$ for $x>0$ is

$$
\begin{aligned}
& \left(\mathcal { D } _ { \eta , \sigma } ^ { - } z ^ { - \lambda \Gamma } I _ { p , q } ^ { m , n } \left[c z^{-\mu}\right.\right. \\
& \left.\left.\quad \begin{array}{c}
\left(a_{1}, \sigma_{1} ; A_{1}: y\right),\left(a_{j}, \sigma_{j} ; A_{j}\right)_{2, p} \\
\left(b_{j}, \rho_{j} ; B_{j}\right)_{1, q}
\end{array}\right]\right)(x) \\
& =x^{-\lambda \Gamma} I_{p+1, q+1}^{m, n+1}\left[c x^{-\mu} \mid \begin{array}{c}
\left(a_{1}, \sigma_{1} ; A_{1}: y\right),(1-\sigma-\eta-\lambda, \mu ; 1),\left(a_{j}, \sigma_{j} ; A_{j}\right)_{2, p} \\
\left(b_{j}, \rho_{j} ; B_{j}\right)_{1, q},(1-\eta-\lambda, \mu ; 1)
\end{array}\right],
\end{aligned}
$$

provided every member in (63) does exist.

\section{The Caputo-type fractional differentiation of incomplete l-functions}

For $\sigma, \rho, \eta \in \mathbb{C}, x>0$ along with $\Re(\sigma)>0$, we characterize the respective Caputo fractional differential operators of left-hand and right-hand type related to the Gauss hypergeometric function as follows (see [26]):

$$
\left({ }^{c} \mathcal{D}_{0+}^{\sigma, \rho, \eta} f\right)(x)=\left(\mathcal{I}_{0+}^{-\sigma+[\Re(\sigma)]+1,-\rho-[\Re(\sigma)]-1, \sigma+\eta-[\Re(\sigma)]-1} f^{([\Re(\sigma)]+1)}\right)(x)
$$


and

$$
\left({ }^{c} \mathcal{D}_{-}^{\sigma, \rho, \eta} f\right)(x)=(-1)^{[\Re(\sigma)]+1}\left(\mathcal{I}_{-}^{-\sigma+[\Re(\sigma)]+1,-\rho-[\Re(\sigma)]-1, \sigma+\eta} f^{([\Re(\sigma)]+1)}\right)(x),
$$

where $f^{(n)}$ stands for the $n$th order derivative pertaining to $f$. The functional relationship within the MSM fractional order derivative of Caputo-type and MSM fractional derivative is similar to that between the Caputo fractional order derivative and fractional derivative of Riemann-Liouville type.

Considering $\sigma, \sigma^{\prime}, \rho, \rho^{\prime}, \eta \in \mathbb{C}, x>0$ together with $\mathfrak{i}(\eta)>0$, the respective left-hand sided and right-hand sided Caputo-type MSM fractional differential operators, related to third Appell function, are defined as

$$
\left({ }^{c} \mathcal{D}_{0+}^{\sigma, \sigma^{\prime}, \rho, \rho^{\prime}, \eta} f\right)(x)=\left(\mathcal{I}_{0+}^{-\sigma^{\prime},-\sigma,-\rho^{\prime}+\alpha,-\rho,-\eta+\alpha} f^{(\alpha)}\right)(x)
$$

and

$$
\left({ }^{c} \mathcal{D}_{-}^{\sigma, \sigma^{\prime}, \rho, \rho^{\prime}, \eta} f\right)(x)=(-1)^{\alpha}\left(\mathcal{I}_{-}^{-\sigma^{\prime},-\sigma,-\rho^{\prime},-\rho+\alpha,-\eta+\alpha} f^{(\alpha)}\right)(x),
$$

where $\alpha=[\Re(\eta)]+1$.

The fractional operators (66) and (67) are connected to (64) and (65) as follows:

$$
\left({ }^{c} \mathcal{D}_{0+}^{0, \sigma^{\prime}, \rho, \rho^{\prime}, \eta} f\right)(x)=\left({ }^{c} \mathcal{D}_{0+}^{\eta, \sigma^{\prime}-\eta, \rho^{\prime}-\eta} f\right)(x)
$$

and

$$
\left({ }^{c} \mathcal{D}_{-}^{0, \sigma^{\prime}, \rho, \rho^{\prime}, \eta} f\right)(x)=\left({ }^{c} \mathcal{D}_{-}^{\eta, \sigma^{\prime}-\eta, \rho^{\prime}-\eta} f\right)(x) .
$$

Now, we derive the Caputo-type MSM fractional order derivatives referring to the incomplete $I$-functions. The following are well-known results and will be used in subsequent theorems (see [13]).

Lemma 4.1 Let $\sigma, \sigma^{\prime}, \rho, \rho^{\prime}, \eta, \lambda \in \mathbb{C}$ and $\kappa=[\Re(\eta)]+1$.

(a) If $\Re(\lambda)-\kappa>\max \left\{0, \Re(-\sigma+\rho), \mathfrak{R}\left(-\sigma-\sigma^{\prime}-\rho^{\prime}+\eta\right)\right\}$, then

$$
\begin{aligned}
\left({ }^{c} \mathcal{D}_{0+}^{\sigma, \sigma^{\prime}, \rho, \rho^{\prime}, \eta} t^{\lambda-1}\right)(x)= & x^{\sigma+\sigma^{\prime}-\eta+\lambda-1} \\
& \times \frac{\Gamma(\lambda) \Gamma(\sigma-\rho+\lambda-\kappa) \Gamma\left(\sigma+\sigma^{\prime}+\rho^{\prime}-\eta+\lambda-\kappa\right)}{\Gamma(-\rho+\lambda-\kappa) \Gamma\left(\sigma+\sigma^{\prime}-\eta+\lambda\right) \Gamma\left(\sigma+\rho^{\prime}-\eta+\lambda-\kappa\right)} .
\end{aligned}
$$

(b) If $\Re(\lambda)+\kappa>\max \left\{\Re\left(-\rho^{\prime}\right), \mathfrak{R}\left(\sigma^{\prime}+\rho-\eta\right), \mathfrak{R}\left(\sigma+\sigma^{\prime}-\eta\right)+[\Re(\eta)]+1\right\}$, then

$$
\begin{aligned}
\left({ }^{c} \mathcal{D}_{-}^{\sigma, \sigma^{\prime}, \rho, \rho^{\prime}, \eta} t^{-\lambda}\right)(x)= & x^{\sigma+\sigma^{\prime}-\eta-\lambda} \\
& \times \frac{\Gamma\left(\rho^{\prime}+\lambda+\kappa\right) \Gamma\left(-\sigma-\sigma^{\prime}+\eta+\lambda\right) \Gamma\left(-\sigma^{\prime}-\rho+\eta+\lambda+\kappa\right)}{\Gamma(\lambda) \Gamma\left(-\sigma^{\prime}+\rho^{\prime}+\lambda+\kappa\right) \Gamma\left(-\sigma-\sigma^{\prime}-\rho+\eta+\lambda+\kappa\right)} .
\end{aligned}
$$


First, we give the left-hand sided Caputo-type MSM fractional derivative of the incomplete $I$-functions.

Theorem 4.2 Let $\sigma, \sigma^{\prime}, \rho, \rho^{\prime}, \eta, \lambda, a \in \mathbb{C}, \kappa=[\Re(\eta)]+1$ be such that $\mu>0$ and $\Re(\lambda)-\kappa>$ $\max \left\{0, \Re(-\sigma+\rho), \Re\left(-\sigma-\sigma^{\prime}-\rho^{\prime}+\eta\right)\right\}$. Then, for $x>0$,

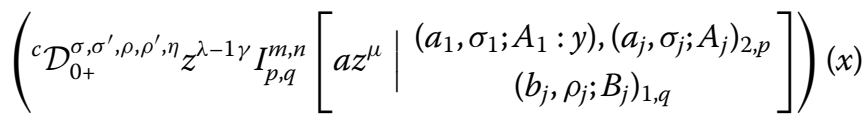

$$
\begin{aligned}
& =x^{\sigma+\sigma^{\prime}-\eta+\lambda-1} \\
& \times^{\gamma} I_{p+3, q+3}^{m, n+3}\left[a x^{\mu} \mid \begin{array}{c}
\left(a_{1}, \sigma_{1} ; A_{1}: y\right),(1-\lambda, \mu ; 1),(1-\sigma+\rho-\lambda+\kappa, \mu ; 1), \\
\left(b_{j}, \rho_{j} ; B_{j}\right)_{1, q},(1+\rho-\lambda+\kappa, \mu ; 1),
\end{array}\right. \\
& \left.\begin{array}{c}
\left(1-\sigma-\sigma^{\prime}-\rho^{\prime}+\eta-\lambda+\kappa, \mu ; 1\right),\left(a_{j}, \sigma_{j} ; A_{j}\right)_{2, p} \\
\left(1-\sigma-\sigma^{\prime}+\eta-\lambda, \mu ; 1\right),\left(1-\sigma-\rho^{\prime}+\eta-\lambda+\kappa, \mu ; 1\right)
\end{array}\right],
\end{aligned}
$$

provided every member in (72) does exist.

Proof The LHS of (72) is given by

$$
\left({ }^{c} \mathcal{D}_{0+}^{\sigma, \sigma^{\prime}, \rho, \rho^{\prime}, \eta}\left(z^{\lambda-1} \frac{1}{2 \pi i} \int_{\$} \phi(s, y) a^{s} z^{\mu s} d s\right)\right)(x),
$$

where $\phi(s, y)$ is given in (3). Interchanging the order of integration in the above equation yields

$$
\frac{1}{2 \pi i} \int_{\$} \phi(s, y) a^{s}\left({ }^{c} \mathcal{D}_{0+}^{\sigma, \sigma^{\prime}, \rho, \rho^{\prime}, \eta} z^{\lambda+\mu s-1}\right)(x) d s,
$$

using the results (70) and (3), we get the RHS of (72).

The properties given below are immediate consequences of definitions (1), (2), and (70), and consequently they are provided without detailed proof as follows.

Theorem 4.3 Let $\sigma, \sigma^{\prime}, \rho, \rho^{\prime}, \eta, \lambda, a \in \mathbb{C}, \kappa=[\Re(\eta)]+1$ be such that $\mu>0$ and $\Re(\lambda)-\kappa>$ $\max \left\{0, \Re(-\sigma+\rho), \Re\left(-\sigma-\sigma^{\prime}-\rho^{\prime}+\eta\right)\right\}$. Then, for $x>0$,

$$
\begin{aligned}
& \left({ }^{c} \mathcal{D}_{0+}^{\sigma, \sigma^{\prime}, \rho, \rho^{\prime}, \eta} z^{\lambda-1 \Gamma} I_{p, q}^{m, n}\left[a z^{\mu} \mid \begin{array}{c}
\left(a_{1}, \sigma_{1} ; A_{1}: y\right),\left(a_{j}, \sigma_{j} ; A_{j}\right)_{2, p} \\
\left(b_{j}, \rho_{j} ; B_{j}\right)_{1, q}
\end{array}\right]\right)(x) \\
& =x^{\sigma+\sigma^{\prime}-\eta+\lambda-1} \\
& \times{ }^{\Gamma} I_{p+3, q+3}^{m, n+3}\left[a x^{\mu} \mid \begin{array}{c}
\left(a_{1}, \sigma_{1} ; A_{1}: y\right),(1-\lambda, \mu ; 1),(1-\sigma+\rho-\lambda+\kappa, \mu ; 1), \\
\left(b_{j}, \rho_{j} ; B_{j}\right)_{1, q},(1+\rho-\lambda+\kappa, \mu ; 1),
\end{array}\right. \\
& \left.\begin{array}{c}
\left(1-\sigma-\sigma^{\prime}-\rho^{\prime}+\eta-\lambda+\kappa, \mu ; 1\right),\left(a_{j}, \sigma_{j} ; A_{j}\right)_{2, p} \\
\left(1-\sigma-\sigma^{\prime}+\eta-\lambda, \mu ; 1\right),\left(1-\sigma-\rho^{\prime}+\eta-\lambda+\kappa, \mu ; 1\right)
\end{array}\right],
\end{aligned}
$$

provided every member in (75) does exist. 
Corollary 25 Let $\sigma, \rho, \eta, \lambda, a \in \mathbb{C}, \kappa=[\Re(\sigma)]+1$ be such that $\mu>0$ and $\Re(\lambda)-\kappa>$ $\max \{0, \Re(-\sigma-\rho-\eta)\}$. Then left-hand sided generalized Caputo fractional differentiation ${ }^{c} \mathcal{D}_{0+}^{\sigma, \rho, \eta}$ of the incomplete I-function is given for $x>0$ by

$$
\begin{array}{r}
\left({ }^{c} \mathcal{D}_{0+}^{\sigma, \rho, \eta} z^{\lambda-1 \gamma} I_{p, q}^{m, n}\left[a z^{\mu} \mid \begin{array}{c}
\left(a_{1}, \sigma_{1} ; A_{1}: y\right),\left(a_{j}, \sigma_{j} ; A_{j}\right)_{2, p} \\
\left(b_{j}, \rho_{j} ; B_{j}\right)_{1, q}
\end{array}\right]\right)(x) \\
=x^{\rho+\lambda-1 \gamma} I_{p+2, q+2}^{m, n+2}\left[\begin{array}{l}
a x^{\mu} \mid \begin{array}{c}
\left(a_{1}, \sigma_{1} ; A_{1}: y\right),(1-\lambda, \mu ; 1), \\
\left(b_{j}, \rho_{j} ; B_{j}\right)_{1, q},(1-\rho-\lambda, \mu ; 1),
\end{array} \\
(1-\sigma-\rho-\eta-\lambda+\kappa, \mu ; 1),\left(a_{j}, \sigma_{j} ; A_{j}\right)_{2, p} \\
(1-\eta-\lambda+\kappa, \mu ; 1)
\end{array}\right],
\end{array}
$$

provided every member in (76) does exist.

Corollary 26 Let $\sigma, \rho, \eta, \lambda, a \in \mathbb{C}, \kappa=[\Re(\sigma)]+1$ be such that $\mu>0$ and $\Re(\lambda)-\kappa>$ $\max \{0, \Re(-\sigma-\rho-\eta)\}$. Then left-hand sided generalized Caputo fractional differentiation ${ }^{c} \mathcal{D}_{0+}^{\sigma, \rho, \eta}$ of the incomplete I-function is given for $x>0$ by

$$
\begin{array}{r}
\left({ } ^ { c } \mathcal { D } _ { 0 + } ^ { \sigma , \rho , \eta } z ^ { \lambda - 1 \Gamma } I _ { p , q } ^ { m , n } \left[a z^{\mu}\right.\right. \\
\left.\left.\begin{array}{c}
\left(a_{1}, \sigma_{1} ; A_{1}: y\right),\left(a_{j}, \sigma_{j} ; A_{j}\right)_{2, p} \\
\left(b_{j}, \rho_{j} ; B_{j}\right)_{1, q}
\end{array}\right]\right)(x) \\
=x^{\rho+\lambda-1 \Gamma} I_{p+2, q+2}^{m, n+2}\left[\begin{array}{c|c}
a x^{\mu} \mid \begin{array}{c}
\left(a_{1}, \sigma_{1} ; A_{1}: y\right),(1-\lambda, \mu ; 1), \\
\left(b_{j}, \rho_{j} ; B_{j}\right)_{1, q},(1-\rho-\lambda, \mu ; 1),
\end{array} \\
(1-\sigma-\rho-\eta-\lambda+\kappa, \mu ; 1),\left(a_{j}, \sigma_{j} ; A_{j}\right)_{2, p} \\
(1-\eta-\lambda+\kappa, \mu ; 1)
\end{array}\right],
\end{array}
$$

provided every member in (77) does exist.

Corollary 27 Let $\sigma, \eta, \lambda, a \in \mathbb{C}, \kappa=[\Re(\sigma)]+1$ be such that $\mu>0$ and $\Re(\lambda)-\kappa>$ $\max \{0, \Re(-\sigma-\eta)\}$. Then left-hand sided Caputo-type Erdélyi-Kober fractional differentiation ${ }^{c} \mathcal{D}_{\eta, \sigma}^{+}\left(=\mathcal{D}_{0+}^{\sigma, 0, \eta}\right)$ of the incomplete I-function is given for $x>0$ by

$$
\begin{aligned}
& \left.\left(\begin{array}{c}
{ }^{c} \mathcal{D}_{\eta, \sigma}^{+} z^{\lambda-1 \gamma} I_{p, q}^{m, n} \\
=
\end{array} \mid \begin{array}{c}
a z^{\mu} \\
\left.\left(b_{j}, \rho_{j} ; B_{j}\right)_{1, q}, \sigma_{1} ; A_{1}: y\right),\left(a_{j}, \sigma_{j} ; A_{j}\right)_{2, p}
\end{array}\right]\right)(x) \\
& \quad \times{ }^{\gamma} I_{p+1, q+1}^{m, n+1}\left[a x^{\mu}\right. \\
& \left.\begin{array}{c}
\left(a_{1}, \sigma_{1} ; A_{1}: y\right),(1-\sigma-\eta-\lambda+\kappa, \mu ; 1),\left(a_{j}, \sigma_{j} ; A_{j}\right)_{2, p} \\
\left(b_{j}, \rho_{j} ; B_{j}\right)_{1, q},(1-\eta-\lambda+\kappa, \mu ; 1)
\end{array}\right],
\end{aligned}
$$

provided every member in (78) does exist.

Corollary 28 Let $\sigma, \eta, \lambda, a \in \mathbb{C}, \kappa=[\Re(\sigma)]+1$ be such that $\mu>0$ and $\Re(\lambda)-\kappa>$ $\max \{0, \Re(-\sigma-\eta)\}$. Then left-hand sided Caputo-type Erdélyi-Kober fractional differen- 
tiation ${ }^{c} \mathcal{D}_{\eta, \sigma}^{+}\left(=\mathcal{D}_{0+}^{\sigma, 0, \eta}\right)$ of the incomplete I-function is given for $x>0$ by

$$
\begin{aligned}
& \left(\begin{array}{c}
{ }^{c} \mathcal{D}_{\eta, \sigma}^{+} z^{\lambda-1 \Gamma} I_{p, q}^{m, n}\left[a z^{\mu}\right. \\
=x^{\lambda-1} \\
\left.\left(b_{j}, \rho_{j} ; B_{j}\right)_{1, q}, \sigma_{1} ; A_{1}: y\right),\left(a_{j}, \sigma_{j} ; A_{j}\right)_{2, p} \\
(x))(x)
\end{array}\right) \\
& \quad \times{ }^{\Gamma} I_{p+1, q+1}^{m, n+1}\left[\begin{array}{c}
a x^{\mu} \\
\left(a_{1}, \sigma_{1} ; A_{1}: y\right),(1-\sigma-\eta-\lambda+\kappa, \mu ; 1),\left(a_{j}, \sigma_{j} ; A_{j}\right)_{2, p} \\
\left(b_{j}, \rho_{j} ; B_{j}\right)_{1, q},(1-\eta-\lambda+\kappa, \mu ; 1)
\end{array}\right],
\end{aligned}
$$

provided every member in (79) does exist.

Lastly, we represent the right-hand sided Caputo-type MSM fractional derivative of the incomplete $I$-functions.

Theorem 4.4 Let $\sigma, \sigma^{\prime}, \rho, \rho^{\prime}, \eta, \lambda, a \in \mathbb{C}, \kappa=[\Re(\eta)]+1$ be such that $\mu>0$ and $\Re(\lambda)+\kappa>$ $\max \left\{\Re\left(-\rho^{\prime}\right), \mathfrak{R}\left(\sigma^{\prime}+\rho-\eta\right), \mathfrak{i}\left(\sigma+\sigma^{\prime}-\eta\right)+\kappa\right\}$. Then, for $x>0$,

$$
\begin{aligned}
& \left({ }^{c} \mathcal{D}_{-}^{\sigma, \sigma^{\prime}, \rho, \rho^{\prime}, \eta} z^{-\lambda \gamma} I_{p, q}^{m, n}\left[a z^{-\mu} \mid \begin{array}{c}
\left(a_{1}, \sigma_{1} ; A_{1}: y\right),\left(a_{j}, \sigma_{j} ; A_{j}\right)_{2, p} \\
\left(b_{j}, \rho_{j} ; B_{j}\right)_{1, q}
\end{array}\right]\right)(x) \\
& =x^{\sigma+\sigma^{\prime}-\eta-\lambda} \\
& \times{ }^{\gamma} I_{p+3, q+3}^{m, n+3}\left[a x^{-\mu} \mid \begin{array}{c}
\left(a_{1}, \sigma_{1} ; A_{1}: y\right),\left(1-\rho^{\prime}-\lambda-\kappa, \mu ; 1\right),\left(1+\sigma+\sigma^{\prime}-\eta-\lambda, \mu ; 1\right) \\
\left(b_{j}, \rho_{j} ; B_{j}\right)_{1, q},(1-\lambda, \mu ; 1),
\end{array}\right. \\
& \left.\begin{array}{c}
\left(1+\sigma^{\prime}+\rho-\eta-\lambda-\kappa, \mu ; 1\right),\left(a_{j}, \sigma_{j} ; A_{j}\right)_{2, p} \\
\left(1+\sigma^{\prime}-\rho^{\prime}-\lambda-\kappa, \mu ; 1\right),\left(1+\sigma+\sigma^{\prime}+\rho-\eta-\lambda-\kappa, \mu ; 1\right)
\end{array}\right],
\end{aligned}
$$

provided every member in (80) does exist.

Proof The LHS of (80) is given by

$$
\left({ }^{c} \mathcal{D}_{-}^{\sigma, \sigma^{\prime}, \rho, \rho^{\prime}, \eta}\left(z^{-\lambda} \frac{1}{2 \pi i} \int_{\$} \phi(s, y) a^{s} z^{-\mu s} d s\right)\right)(x),
$$

where $\phi(s, y)$ is given in (3). Interchanging the order of integration in the above equation yields

$$
\frac{1}{2 \pi i} \int_{\$} \phi(s, y) a^{s}\left({ }^{c} \mathcal{D}_{-}^{\sigma, \sigma^{\prime}, \rho, \rho^{\prime}, \eta} z^{-(\lambda+\mu s)}\right)(x) d s,
$$

using the results (71) and (3), we get the RHS of (80).

Theorem 4.5 Let $\sigma, \sigma^{\prime}, \rho, \rho^{\prime}, \eta, \lambda, a \in \mathbb{C}, \kappa=[\Re(\eta)]+1$ be such that $\mu>0$ and $\Re(\lambda)+\kappa>$ $\max \left\{\Re\left(-\rho^{\prime}\right), \mathfrak{R}\left(\sigma^{\prime}+\rho-\eta\right), \mathfrak{R}\left(\sigma+\sigma^{\prime}-\eta\right)+\kappa\right\}$. Then, for $x>0$,

$$
\begin{aligned}
& \left.\left(\begin{array}{l}
{ }^{c} \mathcal{D}_{-}^{\sigma, \sigma^{\prime}, \rho, \rho^{\prime}, \eta} z^{-\lambda \Gamma} I_{p, q}^{m, n}\left[a z^{-\mu}\right. \\
\left(a_{1}, \sigma_{1} ; A_{1}: y\right),\left(a_{j}, \sigma_{j} ; A_{j}\right)_{2, p} \\
\left(b_{j}, \rho_{j} ; B_{j}\right)_{1, q}
\end{array}\right]\right)(x) \\
& =x^{\sigma+\sigma^{\prime}-\eta-\lambda}
\end{aligned}
$$




$$
\left.\begin{array}{rl}
\times{ }^{\Gamma} I_{p+3, q+3}^{m, n+3}\left[a x^{-\mu} \mid \begin{array}{c}
\left(a_{1}, \sigma_{1} ; A_{1}: y\right),\left(1-\rho^{\prime}-\lambda-\kappa, \mu ; 1\right),\left(1+\sigma+\sigma^{\prime}-\eta-\lambda, \mu ; 1\right) \\
\left(b_{j}, \rho_{j} ; B_{j}\right)_{1, q},(1-\lambda, \mu ; 1)
\end{array}\right. \\
\left(1+\sigma^{\prime}+\rho-\eta-\lambda-\kappa, \mu ; 1\right),\left(a_{j}, \sigma_{j} ; A_{j}\right)_{2, p} \\
\left(1+\sigma^{\prime}-\rho^{\prime}-\lambda-\kappa, \mu ; 1\right),\left(1+\sigma+\sigma^{\prime}+\rho-\eta-\lambda-\kappa, \mu ; 1\right)
\end{array}\right], \quad \text { (83) }
$$

provided every member in (83) does exist.

Corollary 29 Let $\sigma, \rho, \eta, \lambda, a \in \mathbb{C}, \kappa=[\Re(\sigma)]+1$ be such that $\mu>0$ and $\Re(\lambda)+\kappa>$ $\max \{\Re(-\sigma-\eta), \mathfrak{R}(\rho)+\kappa\}$. Then the right-hand sided generalized Caputo fractional derivative ${ }^{c} \mathcal{D}_{-}^{\sigma, \rho, \eta}$ of the incomplete I-function is given for $x>0$ by

$$
\begin{gathered}
\left({ }^{c} \mathcal{D}_{-}^{\sigma, \rho, \eta} z^{-\lambda \gamma} I_{p, q}^{m, n}\left[a z^{-\mu} \mid \begin{array}{c}
\left(a_{1}, \sigma_{1} ; A_{1}: y\right),\left(a_{j}, \sigma_{j} ; A_{j}\right)_{2, p} \\
\left(b_{j}, \rho_{j} ; B_{j}\right)_{1, q}
\end{array}\right]\right)(x) \\
=x^{\rho-\lambda \gamma} I_{p+2, q+2}^{m, n+2}\left[\begin{array}{c|c}
a x^{-\mu} & \left(\begin{array}{c}
\left(a_{1}, \sigma_{1} ; A_{1}: y\right),(1-\sigma-\eta-\lambda-\kappa, \mu ; 1), \\
\left(b_{j}, \rho_{j} ; B_{j}\right)_{1, q},(1-\lambda, \mu ; 1),
\end{array}\right. \\
(1+\rho-\lambda, \mu ; 1),\left(a_{j}, \sigma_{j} ; A_{j}\right)_{2, p} \\
(1+\rho-\eta-\lambda-\kappa, \mu ; 1)
\end{array}\right]
\end{gathered}
$$

provided every member in (84) does exist.

Corollary 30 Let $\sigma, \rho, \eta, \lambda, a \in \mathbb{C}, \kappa=[\Re(\sigma)]+1$ be such that $\mu>0$ and $\Re(\lambda)+\kappa>$ $\max \{\Re(-\sigma-\eta), \Re(\rho)+\kappa\}$. Then the right-hand sided generalized Caputo fractional derivative ${ }^{c} \mathcal{D}_{-}^{\sigma, \rho, \eta}$ of the incomplete I-function is given for $x>0$ by

$$
\begin{gathered}
\left({ }^{c} \mathcal{D}_{-}^{\sigma, \rho, \eta} z^{-\lambda \Gamma} I_{p, q}^{m, n}\left[a z^{-\mu} \mid \begin{array}{c}
\left(a_{1}, \sigma_{1} ; A_{1}: y\right),\left(a_{j}, \sigma_{j} ; A_{j}\right)_{2, p} \\
\left(b_{j}, \rho_{j} ; B_{j}\right)_{1, q}
\end{array}\right]\right)(x) \\
=x^{\rho-\lambda \Gamma} I_{p+2, q+2}^{m, n+2}\left[\begin{array}{c}
a x^{-\mu} \mid \begin{array}{c}
\left(a_{1}, \sigma_{1} ; A_{1}: y\right),(1-\sigma-\eta-\lambda-\kappa, \mu ; 1) \\
\left(b_{j}, \rho_{j} ; B_{j}\right)_{1, q},(1-\lambda, \mu ; 1),
\end{array} \\
(1+\rho-\lambda, \mu ; 1),\left(a_{j}, \sigma_{j} ; A_{j}\right)_{2, p} \\
(1+\rho-\eta-\lambda-\kappa, \mu ; 1)
\end{array}\right],
\end{gathered}
$$

provided every member in (85) does exist.

Corollary 31 Let $\sigma, \eta, \lambda, a \in \mathbb{C}, \kappa=[\Re(\sigma)]+1$ be such that $\mu>0$ and $\Re(\lambda)+\kappa>$ $\max \{\Re(-\sigma-\eta), \kappa\}$. Then the right-hand sided Caputo-type Erdélyi-Kober fractional derivative ${ }^{c} \mathcal{D}_{\eta, \sigma}^{-}\left(={ }^{c} \mathcal{D}_{-}^{\sigma, \rho, \eta}\right)$ of the incomplete I-function is given for $x>0$ by

$$
\begin{aligned}
& \left({ }^{c} \mathcal{D}_{\eta, \sigma}^{-} z^{-\lambda \gamma} I_{p, q}^{m, n}\left[a z^{-\mu} \mid \begin{array}{c}
\left(a_{1}, \sigma_{1} ; A_{1}: y\right),\left(a_{j}, \sigma_{j} ; A_{j}\right)_{2, p} \\
\left(b_{j}, \rho_{j} ; B_{j}\right)_{1, q}
\end{array}\right]\right)(x) \\
& =x^{-\lambda} \\
& \times{ }^{\gamma} I_{p+1, q+1}^{m, n+1}\left[a x^{-\mu} \mid \begin{array}{c}
\left(a_{1}, \sigma_{1} ; A_{1}: y\right),(1-\sigma-\eta-\lambda-\kappa, \mu ; 1),\left(a_{j}, \sigma_{j} ; A_{j}\right)_{2, p} \\
\left(b_{j}, \rho_{j} ; B_{j}\right)_{1, q},(1-\eta-\lambda-\kappa, \mu ; 1)
\end{array}\right],
\end{aligned}
$$

provided every member in (86) does exist. 
Corollary 32 Let $\sigma, \eta, \lambda, a \in \mathbb{C}, \kappa=[\Re(\sigma)]+1$ be such that $\mu>0$ and $\Re(\lambda)+\kappa>$ $\max \{\Re(-\sigma-\eta), \kappa\}$. Then the right-hand sided Caputo-type Erdélyi-Kober fractional derivative ${ }^{c} \mathcal{D}_{\eta, \sigma}^{-}\left(={ }^{c} \mathcal{D}_{-}^{\sigma, \rho, \eta}\right)$ of the incomplete I-function is given for $x>0$ by

$$
\begin{aligned}
& \left.\left(\begin{array}{c}
{ }^{c} \mathcal{D}_{\eta, \sigma}^{-} z^{-\lambda \Gamma} I_{p, q}^{m, n}\left[a z^{-\mu}\right. \\
\left(a_{1}, \sigma_{1} ; A_{1}: y\right),\left(a_{j}, \sigma_{j} ; A_{j}\right)_{2, p} \\
\left(b_{j}, \rho_{j} ; B_{j}\right)_{1, q}
\end{array}\right]\right)(x) \\
& =x^{-\lambda} \\
& \quad \times{ }^{\Gamma} I_{p+1, q+1}^{m, n+1}\left[a x^{-\mu} \mid \begin{array}{c}
\left(a_{1}, \sigma_{1} ; A_{1}: y\right),(1-\sigma-\eta-\lambda-\kappa, \mu ; 1),\left(a_{j}, \sigma_{j} ; A_{j}\right)_{2, p} \\
\left(b_{j}, \rho_{j} ; B_{j}\right)_{1, q},(1-\eta-\lambda-\kappa, \mu ; 1)
\end{array}\right],
\end{aligned}
$$

provided every member in (87) does exist.

\section{Special cases and concluding remarks}

By specializing the parameters in definition (2), we obtain the following functions as special cases of the incomplete $I$-function ${ }^{\Gamma} I_{p, q}^{m, n}(z)$ :

(i) Incomplete $\bar{I}$-function ${ }^{\Gamma} \bar{I}_{p, q}^{m, n}(z)$ : if we set $B_{j}(j=1, \ldots, m)=1$ in (2), then we obtain

$$
\begin{aligned}
\Gamma_{\bar{I}} \bar{I}_{p, q}^{m, n}(z) & =\Gamma_{\bar{I}}^{m, n}\left[z \mid \begin{array}{l}
\left(a_{1}, \sigma_{1} ; A_{1}: x\right),\left(a_{j}, \sigma_{j} ; A_{j}\right)_{2, p} \\
\left(b_{j}, \rho_{j} ; 1\right)_{1, m},\left(b_{j}, \rho_{j} ; B_{j}\right)_{m+1, q}
\end{array}\right] \\
& =\frac{1}{2 \pi i} \int_{\$} \bar{\phi}(s, x) z^{s} d s,
\end{aligned}
$$

where

$$
\bar{\phi}(s, x)=\frac{\left\{\gamma\left(1-a_{1}+\sigma_{1} s, x\right)\right\}^{A_{1}} \prod_{j=1}^{m} \Gamma\left(b_{j}-\rho_{j} s\right) \prod_{j=2}^{n}\left\{\Gamma\left(1-a_{j}+\sigma_{j} s\right)\right\}^{A_{j}}}{\prod_{j=n+1}^{p}\left\{\Gamma\left(a_{j}-\sigma_{j} s\right)\right\}^{A_{j}} \prod_{j=m+1}^{q}\left\{\Gamma\left(1-b_{j}+\rho_{j} s\right)\right\}^{B_{j}}} .
$$

(ii) Incomplete $\bar{H}$-function $\bar{\Gamma}_{p, q}^{m, n}(z)$ : if we set $B_{j}(j=1, \ldots, m)=1$ and

$A_{j}(j=n+1, \ldots, p)=1$ in (2), then we obtain (see [32])

$$
\begin{aligned}
\bar{\Gamma}_{p, q}^{m, n}(z) & =\Gamma_{\bar{I}, q}^{m, n}\left[z \mid \begin{array}{c}
\left(a_{1}, \sigma_{1} ; A_{1}: x\right),\left(a_{j}, \sigma_{j} ; A_{j}\right)_{2, n},\left(a_{j}, \sigma_{j} ; 1\right)_{n+1, p} \\
\left(b_{j}, \rho_{j} ; 1\right)_{1, m},\left(b_{j}, \rho_{j} ; B_{j}\right)_{m+1, q}
\end{array}\right] \\
& =\bar{\Gamma}_{p, q}^{m, n}\left[z \mid \begin{array}{c}
\left(a_{1}, \sigma_{1} ; A_{1}: x\right),\left(a_{j}, \sigma_{j} ; A_{j}\right)_{2, n},\left(a_{j}, \sigma_{j}\right)_{n+1, p} \\
\left(b_{j}, \rho_{j}\right)_{1, m},\left(b_{j}, \rho_{j} ; B_{j}\right)_{m+1, q}
\end{array}\right] \\
& =\frac{1}{2 \pi i} \int_{\$} \bar{\psi}(s, x) z^{s} d s,
\end{aligned}
$$

where

$$
\bar{\psi}(s, x)=\frac{\left\{\gamma\left(1-a_{1}+\sigma_{1} s, x\right)\right\}^{A_{1}} \prod_{j=1}^{m} \Gamma\left(b_{j}-\rho_{j} s\right) \prod_{j=2}^{n}\left\{\Gamma\left(1-a_{j}+\sigma_{j} s\right)\right\}^{A_{j}}}{\prod_{j=n+1}^{p} \Gamma\left(a_{j}-\sigma_{j} s\right) \prod_{j=m+1}^{q}\left\{\Gamma\left(1-b_{j}+\rho_{j} s\right)\right\}^{B_{j}}} .
$$


(iii) Incomplete $H$-function $\Gamma_{p, q}^{m, n}(z)$ : if we set $B_{j}(j=1, \ldots, q)=1$ and $A_{j}(j=1, \ldots, p)=1$ in (2), then we obtain (see [32])

$$
\begin{aligned}
\Gamma_{p, q}^{m, n}(z) & =\Gamma_{p, q}^{m, n}\left[\begin{array}{c}
\left(a_{1}, \sigma_{1} ; 1: x\right),\left(a_{j}, \sigma_{j} ; 1\right)_{2, p} \\
\left(b_{j}, \rho_{j} ; 1\right)_{1, q}
\end{array}\right] \\
& =\Gamma_{p, q}^{m, n}\left[\begin{array}{c}
\left(a_{1}, \sigma_{1}: x\right),\left(a_{j}, \sigma_{j}\right)_{2, p} \\
\left(b_{j}, \rho_{j}\right)_{1, q}
\end{array}\right] \\
& =\frac{1}{2 \pi i} \int_{\$} \psi(s, x) z^{s} d s,
\end{aligned}
$$

where

$$
\psi(s, x)=\frac{\gamma\left(1-a_{1}+\sigma_{1} s, x\right) \prod_{j=1}^{m} \Gamma\left(b_{j}-\rho_{j} s\right) \prod_{j=2}^{n} \Gamma\left(1-a_{j}+\sigma_{j} s\right)}{\prod_{j=n+1}^{p} \Gamma\left(a_{j}-\sigma_{j} s\right) \prod_{j=m+1}^{q} \Gamma\left(1-b_{j}+\rho_{j} s\right)} .
$$

(iv) Incomplete Fox-Wright function ${ }_{p} \Psi_{q}^{(\Gamma)}(z)$ : if we take the substitutions $z=-z$,

$A_{j}(j=1, \ldots, p)=B_{j}(j=1, \ldots, q)=1, a_{j} \rightarrow\left(1-a_{j}\right)(j=1, \ldots, p)$ and $b_{j} \rightarrow\left(1-b_{j}\right)$ $(j=1, \ldots, q)$ in $(2)$, then we obtain (see [24])

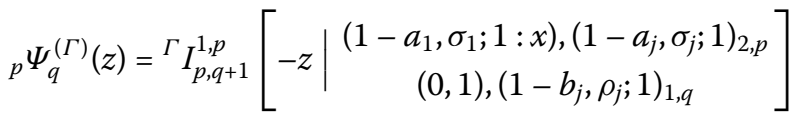

$$
\begin{aligned}
& ={ }_{p} \Psi_{q}^{(\Gamma)}\left[\begin{array}{c}
\left(a_{1}, \sigma_{1}, x\right),\left(a_{j}, \sigma_{j}\right)_{2, p} ; \\
\left(b_{j}, \rho_{j}\right)_{1, q} ;
\end{array}\right] \text {. }
\end{aligned}
$$

Remark 1 Similarly, one can easily obtain another class of incomplete functions as special cases of the incomplete $I$-function $\gamma \bar{I}_{p, q}^{m, n}(z)$.

It is to note that if we use the relations (90) and (92), then one can obtain the fractional calculus results associated with the incomplete $H$-functions and incomplete $\bar{H}$-functions (see [32]) as special cases of our results. Moreover, all the results investigated in this paper, taking into account the decomposition formula (8) (or $y=0$ in the results involving $\left.\Gamma I_{p, q}^{m, n}(z)\right)$, lead to the known results provided earlier by Kataria and Vellaisamy [13].

In the present article, we investigated a number of fractional calculus image formulas involving incomplete $I$-functions associated with the MSM operators. The incomplete $I$-functions generalize $I$-function, $\bar{H}$-function, $H$-function, Meijer $G$-function, hypergeometric function, and many other functions. Additionally, the MSM fractional operators generalize Saigo, Riemann-Liouville, Erdélyi-Kober fractional calculus operators. In consideration of the indicated fact, one can obtain numerous image formulas comprising a class of special functions (see $[14,15,27,30,32])$ as limiting cases of the main outcomes.

Acknowledgements

The authors express their sincere thanks to the referee for his/her careful reading and suggestions that helped to improve this paper.

Funding

Not applicable.

Availability of data and materials

Not applicable. 


\section{Competing interests}

The authors declare that they have no competing interests.

\section{Authors' contributions}

The authors contributed equally and significantly in writing this paper. All authors read and approved the final manuscript.

\section{Author details}

${ }^{1}$ Department of HEAS (Mathematics), Rajasthan Technical University, Kota, India. ${ }^{2}$ Department of Mathematics, Malaviya National Institute of Technology, Jaipur, India. ${ }^{3}$ Department of Mathematics, Cankaya University, Ankara, Turkey. ${ }^{4}$ Institute of Space Sciences, Magurele-Bucharest, Romania. ${ }^{5}$ Department of Medical Research, China Medical University Hospital, China Medical University, Taichung, Taiwan. ${ }^{6}$ Department of Mathematics, College of Science, King Saud University, Riyadh, Saudi Arabia.

\section{Publisher's Note}

Springer Nature remains neutral with regard to jurisdictional claims in published maps and institutional affiliations.

Received: 23 March 2020 Accepted: 25 May 2020 Published online: 04 June 2020

\section{References}

1. Atangana, A., Baleanu, D.: New fractional derivatives with nonlocal and non-singular kernel: theory and application to heat transfer model. Therm. Sci. 20(2), 763-769 (2016)

2. Baleanu, D., Kumar, D., Purohit, S.D.: Generalized fractional integrals of product of two $\mathrm{H}$-functions and a general class of polynomials. Int. J. Comput. Math. 93(8), 1320-1329 (2016)

3. Cattani, C.: A review on harmonic wavelets and their fractional extension. J. Adv. Eng. Comput. 2(4), 224-238 (2018)

4. Cattani, C., Rushchitskii, Y.Y.: Cubically nonlinear elastic waves: wave equations and methods of analysis. Int. Appl. Mech. 39, 1115-1145 (2003)

5. Dizicheh, A.K., Salahshour, S., Ahmadian, A., Baleanu, D.: A novel algorithm based on the Legendre wavelets spectral technique for solving the Lane-Emden equations. Appl. Numer. Math. 153, 443-456 (2020)

6. Fox, C.: The $G$ and $H$-functions as symmetrical Fourier kernels. Trans. Am. Math. Soc. 98, 395-429 (1961)

7. Gao, W., Veeresha, P., Prakasha, D.G., Baskonus, H.M., Yel, G.: A powerful approach for fractional Drinfeld-Sokolov-Wilson equation with Mittag-Leffler law. Alex. Eng. J. 58(4), 1301-1311 (2019)

8. Gao, W., Yel, G., Baskonus, H.M., Cattani, C.: Complex solitons in the conformable (2+1)-dimensional Ablowitz-Kaup-Newell-Segur equation. AlMS Math. 5(1), 507-521 (2020)

9. Ghanbari, B., Atangana, A.: A new application of fractional Atangana-Baleanu derivatives: designing ABC-fractional masks in image processing. Phys. A, Stat. Mech. Appl. 542, Article ID 123516 (2020)

10. Gumah, G., Al-Omari, S., Baleanu, D.: Soft computing technique for a system of fuzzy Volterra integro-differential equations in a Hilbert space. Appl. Numer. Math. 152, 310-322 (2020)

11. Jangid, K., Bhatter, S., Meena, S., Baleanu, D., Purohit, S.D.: Certain classes of the incomplete I-functions and their properties (communicated)

12. Jun, Y.X., Baleanu, D., Lazarevic, M.P., Cajic, M.: Fractal boundary value problems for integral and differential equations with local fractional operators. Therm. Sci. 19(3), 959-966 (2015)

13. Kataria, K.K., Vellaisamy, P.: Some fractional calculus results associated with the l-functions. Matematiche $70(2)$ 173-190 (2015)

14. Kilbas, A.A., Saigo, M.: H-Transforms: Theory and Applications. Analytic Methods and Special Functions. An International Series of Monographs in Mathematics, vol. 9. Chapman \& Hall, Boca Raton (2004)

15. Kilbas, A.A., Srivastava, H.M., Trujillo, J.J.: Theory and Applications of Fractional Differential Equations. North-Holland Mathematical Studies, vol. 204. Elsevier, Amsterdam (2006)

16. Kiryakova, V:: Generalized Fractional Calculus and Applications. Longman, Harlow (1994)

17. Kumar, D., Purohit, S.D., Choi, J.: Generalized fractional integrals involving product of multivariable $H$-function and a general class of polynomials. J. Nonlinear Sci. Appl. 9(1), 8-21 (2016)

18. Kumar, D., Singh, J., Baleanu, D.: A new numerical algorithm for fractional Fitzhugh-Nagumo equation arising in transmission of nerve impulses. Nonlinear Dyn. 91, 307-317 (2018)

19. Marichev, O.I.: Volterra equation of Mellin convolution type with a Horn function in the kernel. Izv. Akad. Nauk SSSR 1, 128-129 (1974)

20. Mathai, A.M., Saxena, R.K., Haubold, H.J.: The H-Function Theory and Applications. Springer, New York (2010)

21. Nisar, K.S., Suthar, D.L., Agarwal, R., Purohit, S.D.: Fractional calculus operators with Appell function kernels applied to Srivastava polynomials and extended Mittag-Leffler function. Adv. Differ. Equ. 2020, Article ID 148 (2020)

22. Nisar, K.S., Suthar, D.L., Bohra, M., Purohit, S.D.: Generalized fractional integral operators pertaining to the product of Srivastava's polynomials and generalized Mathieu series. Mathematics 7(2), Article ID 206 (2019)

23. Panda, S.K., Abdeljawad, T., Ravichandrane, C.: Novel fixed point approach to Atangana-Baleanu fractional and Fredholm integral equations. Alex. Eng. J. (2020) https://doi.org/10.1016/j.aej.2019.12.027

24. Parmar, R.K., Saxena, R.K.: Incomplete extended Hurwitz-Lerch zeta functions and associated properties. Commun. Korean Math. Soc. 32, 287-304 (2017)

25. Purohit, S.D., Suthar, D.L., Kalla, S.L.: Marichev-Saigo-Maeda fractional integration operators of the Bessel function. Matematiche 67(1), 21-32 (2012)

26. Rao, A., Garg, M., Kalla, S.L.: Caputo-type fractional derivative of a hypergeometric integral operator. Kuwait J. Sci. Eng. 37(1A), 15-29 (2010)

27. Rathie, A.K.: A new generalization of generalized hypergeometric functions. Matematiche 52(2), 297-310 (1997)

28. Ravichandran, C., Logeswari, K., Jarad, F.: New results on existence in the framework of Atangana-Baleanu derivative for fractional integro-differential equations. Chaos Solitons Fractals 125, 194-200 (2019) 
29. Saigo, M.: A remark on integral operators involving the Gauss hypergeometric functions. Math. Rep. Kyushu Univ. 11(2), 135-143 (1978)

30. Saigo, M., Maeda, N.: More generalization of fractional calculus. In: Rusev, P., Dimovski, I., Kiryakova, V. (eds.) Trans. Meth. Spec. Func., pp. 386-400 (1998)

31. Singh, J., Kumar, D., Hammouch, Z., Atangana, A.: A fractional epidemiological model for computer viruses pertaining to a new fractional derivative. Appl. Math. Comput. 316, 504-515 (2018)

32. Srivastava, H.M., Saxena, R.K., Parmar, R.K.: Some families of the incomplete $H$-functions and the incomplete $\bar{H}$-functions and associated integral transforms and operators of fractional calculus with applications. Russ. J. Math. Phys. 25(1), 116-138 (2018)

33. Tuan, N.H., Baleanu, D., Thach, T.N., O'Regan, D., Can, N.H.: Final value problem for nonlinear time fractional reaction-diffusion equation with discrete data. J. Comput. Appl. Math. 376(1), Article ID 112883 (2020)

34. Xia, M., Wu, Y.C., Sonia, A.: Exact outage probability of dual-hop CSI-assisted AF relaying over Nakagami-m fading channels. IEEE Trans. Signal Process. 60(10), 5578-5583 (2012)

\section{Submit your manuscript to a SpringerOpen ${ }^{\circ}$ journal and benefit from:}

- Convenient online submission

$\checkmark$ Rigorous peer review

- Open access: articles freely available online

- High visibility within the field

- Retaining the copyright to your article

Submit your next manuscript at $\gg$ springeropen.com 